\title{
Love in the Time of COVID-19: A Multi-Wave Study Examining the Salience of Sexual and Relationship Health During the COVID-19 Pandemic
}

\author{
Agnieszka E. Pollard ${ }^{1} \cdot$ Ronald D. Rogge ${ }^{1}$ (I)
}

Received: 20 December 2020 / Revised: 15 October 2021 / Accepted: 25 October 2021 / Published online: 27 January 2022

(c) The Author(s), under exclusive licence to Springer Science+Business Media, LLC, part of Springer Nature 2021

\begin{abstract}
The current study used Family Systems Theory as a framework to clarify the impact of the COVID-19 pandemic on sexual, romantic, and individual functioning. Specifically, sexual and romantic functioning were modeled as key mechanisms linking COVID-19 related stressors (as predictors) to aspects of individual functioning over time (as outcomes). A sample of 1,241 sexually active adults in relationships (47\% married/engaged) was recruited from March 5 to May 5, 2020: 82\% White, 66\% women, $\mathrm{M}=34$ years old, $58 \%$ heterosexual. All participants completed a baseline survey and 642 participants completed at least one of the six, monthly, follow-up surveys. Multilevel SEM models evaluated the model both at the level of stable between-person differences (i.e., level 2) and at the level of within-person change across time (i.e., level 1). The findings suggested that COVID-19 related stress was predictive of lower sexual, romantic, and individual functioning in both levels of the model. Significant indirect paths supported the proposed mediation at the level of within-person change across time: elevations in COVID-19 stress within specific months predicted corresponding drops in sexual functioning, which in turn predicted corresponding drops in romantic functioning, which in turn predicted corresponding drops in individual wellbeing (highlighting points of intervention). In contrast, at the level of between-person differences, stable levels of sexual and relationship satisfaction across the 6 months of the study were not associated with stable levels of COVID-19 stressors (representing sources of resilience that promoted well-being) and stable levels of stress from social isolation predicted stably higher amounts of communicating affection to one's loved ones (suggesting a need for affiliation in the face of chronic stress) whereas stable difficulties with orgasms were linked to stable irritability toward partners and depressive symptoms. Multigroup analyses suggested that the findings generalized across gender, age, race/ethnicity, sexual orientation, relationship stage, and cohabitation groups. Spillover effects within a Family Systems Theory framework clarify how upheaval of the COVID-19 pandemic could have impacted sexual, romantic, and individual functioning in a process-oriented framework, highlighting sources of resilience (sexual satisfaction, communicating affection) and risk (orgasm difficulties).
\end{abstract}

Keywords COVID-19 $\cdot$ SARS-CoV-2 virus $\cdot$ Sexual functioning $\cdot$ Romantic relationship $\cdot$ Orgasm

\section{Introduction}

Although originally discovered in 2019 , the novel severe acute respiratory syndrome coronavirus 2 (SARS-CoV-2) that causes the coronavirus disease (COVID-19) became a worldwide health crisis in early 2020 . The spread of

Ronald D. Rogge

ronald.rogge@rochester.edu

1 Department of Psychology, University of Rochester, 462 Meliora Hall; RC Box 270266, Rochester, NY 14627-0266, USA
COVID-19 has prompted a diverse array of public health measures in countries across the globe, including lockdown procedures, social distancing, and universal mask use policies. Given the elevated rates of lethality associated with COVID-19 (e.g., Zhou et al., 2020), the highly contagious nature of the virus (e.g., Kucharski et al., 2020) and its ability to spread within asymptomatic individuals (e.g., Arons et al., 2020), public health efforts have yielded varied results. Thus, cases have reached 239 million world wide with an associated 4.8 million deaths (New York Times, 2021).

Within the United States, social distancing and mask wearing guidelines were never universally applied across all states, and became increasingly politicized issues within 
the 2020 US presidential election. As a result, the US now leads the world in COVID-19 cases, with over 44.7 million cases and 721,000 deaths (New York Times, 2021). Although recent studies have documented the adverse impact of the COVID-19 pandemic on individual well-being (see Brooks et al., 2020 for a review), fewer studies have examined possible interpersonal mechanisms explaining those links. The current study therefore sought to extend this work by examining sexual functioning (i.e., sexual satisfaction, orgasmic difficulty, sexual desire) and romantic relationship functioning (i.e., relationship satisfaction, irritability with partner, communication of affection) as possible mechanisms linking pandemic related stress to poorer individual functioning, thereby identifying potential sources of risk and resilience to the adverse impact of the COVID-19 pandemic on the lives of individuals.

\section{COVID-19 and Individual/Interpersonal Functioning}

COVID-19 and Individual Well-being. Although the novel SARS-CoV-2 virus can directly impact physical health via the symptoms of COVID-19, it has also been linked to poorer mental health outcomes (e.g., Brooks et al., 2020). For example, a meta-analytic review of 63,439 individuals from 17 unique studies suggested elevated rates of psychological disorders during the COVID-19 pandemic, with over a third of individuals surveyed meeting criteria for a depressive or anxiety disorder (Salari et al., 2020). In fact, recent evidence suggests that the number of individuals with moderate to severe depressive symptoms tripled during the COVID-19 pandemic (Ettman et al., 2020), and meta-analytic estimates suggest that the prevalence of depression appears to have been 7 times higher during the pandemic than rates observed in 2017 (Bueno-Notivol et al., 2021). Consistent with this, the severity of the COVID-19 crisis within cities in China was linked to lower life satisfaction and well-being (Zhang et al., 2020a).

COVID-19 and Sexual Functioning. Stress associated with the COVID-19 pandemic has also been linked to poorer sexual functioning. For example, significant proportions of individuals living in China reported decreases in sexual desire (22\%) and sexual activity with their partners (41\%) in the early months of the pandemic (Li et al., 2020). Similarly, although many individuals living in Italy perceived no changes in their sexual functioning, $18 \%$ and $26 \%$ of men and women (respectively) experienced drops in sexual desire, with $6 \%$ and $18 \%$ experiencing increased difficulty achieving orgasms, and $6 \%$ and $15 \%$ of reporting drops in sexual satisfaction (Panzeri et al., 2020). Similar declines in sexual health were observed in individuals living in Turkey (Karagöz et al., 2020). Results in Taiwan linked such drops in sexual health to general levels of anxiety during the pandemic and COVID-19 specific worries (Ko et al., 2020). Despite these adverse trends, results have also suggested that the sex lives of some sexual relationships might have improved as a result of the COVID-19 pandemic (e.g., Panzeri et al., 2020). This has led to a potentially more nuanced meta-narrative on how the pandemic might impact the sexual functioning of individuals (for a review and commentary see Döring, 2020), and suggests that vibrant sex lives might serve as a source of resilience.

COVID-19 and Relationship Dynamics. External stressors have been linked to greater conflict and irritability and lower satisfaction within romantic relationships, both crosssectionally (e.g., Falconier et al., 2015) and longitudinally (see Karney \& Bradbury, 1995 for a review). Consistent with this, $31 \%$ of individuals living in China reported notable drops in the quality of their romantic relationships in the early months of the pandemic. Similarly, although $20 \%$ of over 3,100 German participants reported increases in relationship quality during the pandemic, twice that percentage $(40 \%)$ reported notable drops in their relationship satisfaction during the early months of the pandemic (Schmid et al., 2021). Consistent with this, $34 \%$ of US adults reported conflict with their partners related to their COVID-19 health concerns or the continuing quarantine restrictions, and that conflict was linked to lower levels of affection and sex within those relationships (Luetke et al., 2020). As with sexual health, this body of work yields mixed results for the possible impact of the pandemic on romantic functioning, with many individuals experiencing relationship declines during the pandemic, while others experienced a strengthening of their relationships. Thus, these results suggest that romantic relationships could potentially serve as a source of risk or resilience during worldwide crises like the COVID-19 pandemic. Consistent with this, individuals in high quality relationships demonstrated better mental health during the early months of the pandemic than single individuals, whereas individuals in poor quality relationships demonstrated poorer mental health (Pieh et al., 2020).

\section{Family Systems Theory}

Following recent calls for the use of conceptual frameworks to synthesize and integrate research on the COVID-19 pandemic (e.g., Pietromonaco \& Overall, 2020), the current study drew upon Family Systems Theory (Broderick, 1993; Minuchin, 1985) as well as more recently developed theories drawing on that foundation (e.g., transactional family dynamics; Schermerhorn \& Cummings, 2008). These theoretical frameworks conceptualize families as a complex system with multiple levels of functioning (e.g., parent-child dyads, coparent dyads, sibling dyads). These theories then posit that the well-being of any family member is intimately linked to processes and interactions occurring within all of the various levels of functioning or systems/dyads within 
the family. In fact, these theories suggest that dynamics within one subsystem or level of functioning can spillover and impact other areas of functioning. For example, conflict between coparents could affect the well-being and behavior of a child not only by souring the overall dynamic within the family, but also by leading those parents to choose harsher forms of parenting behaviors. Consistent with this, disruptions in the romantic relationship of parents have been shown to spillover into other areas of family and child functioning (e.g., Sturge-Apple et al., 2006).

\section{Applying a Family Systems Conceptual Lens}

The current study modeled how various systems within romantic relationships are interconnected and can spillover into one another to shape the course of a romantic relationship. As the romantic dyad is one of the systems within Family Systems Theory, the application of Family Systems Theory to romantic relationships could be conceptualized as focusing in on a subset of processes and systems within that larger model-specifically focusing on the systems relevant to all couples, regardless of whether they are raising children or not. Within this framework, we posited that sexual, romantic, and individual well-being represent distinct sets of close relationship processes/dynamics that can be conceptualized as different levels of functioning or different systems within the relationship. Thus, a recent decline in sexual functioning for a couple (e.g., lower sexual desire, greater difficulties with orgasms) could spillover into other systems, adversely impacting relationship and individual functioning. ${ }^{1}$

COVID-19 Related Stress as an External Trigger. Although often highly effective at slowing the spread of COVID-19, public health policies involving lockdowns and school and business closures led to massive upheaval in the lives of individuals. These measures left individuals not only worried about the risk of contracting COVID-19, but also

\footnotetext{
1 To move beyond simply examining maladaptive processes and negative outcomes like depressive symptoms, the current study sought to align with a growing body of work on positive psychology. Specifically, the development of scales like the positive affect subscale of the Positive and Negative Affect Scale (PANAS; Watson et al., 1988), the positive affect subscale of the Mood and Anxiety Symptom Questionnaire (MASQ; Watson et al., 1995), the Satisfaction with Life Scale (SLWS; Diener et al., 1985), and the Subjective Vitality Scale (SVS; Ryan \& Frederick, 1997) have allowed researchers to balance the study of psychological distress with the study of well-being. Within previous datasets, the authors have found these measures of global positive outcomes to be strongly correlated (i.e., bivariate correlations above .75) and have more specifically found that among these scales, measures of vitality (focused on positive mood and levels of daily energy) seemed to offer the most unique information above and beyond depressive symptoms (i.e., demonstrated lower collinearity with depression). Thus, the current study assessed depressive symptoms and vitality as the two key markers of individual functioning.
}

stressed due to being cutoff and isolated from their friends and loved ones. Such COVID-19 related stressors have been linked to lower vitality and greater distress (Zhang et al., $2020 \mathrm{~b}$ ), as well as lower sleep quality and greater use of rigid/ inflexible coping responses (Peltz et al., 2020). COVID-19 related stressors have also been linked to feeling like a burden on others and a desire for death (Crasta et al., 2020). Thus, higher rates of anxiety and depression have been reported among those who had social distanced and quarantined than those who have not (Tang et al., 2021), and higher levels of loneliness during the pandemic predicted poorer vitality and psychological adjustment (Arslan, 2021). To build on this previous work, the current study modeled COVID-19 stress and social isolation stress as two key forms of COVID-19 related stress (Fig. 1).

Sexual Functioning as a Set of Proximal Processes Impacted by COVID-19 Stress. Given the links between pandemic-related stress and lower sexual functioning (e.g., Ko et al., 2020), we specifically hypothesized that couples' sexual functioning might represent an emotionally vulnerable aspect of their relationship. As a result, that system might be most immediately impacted by the surge of acute stress and upheaval experienced by individuals as the pandemic broke and lockdown procedures were implemented (see Fig. 1A). Consistent with this, intimacy has been shown to mediate links between daily stress and relationship satisfaction (e.g., Harper et al., 2000). As detailed above, the larger body of findings on the impact of the COVID-19 pandemic on sexual functioning has been somewhat mixed, leading to divergent meta-narratives on the roles of sexual functioning during the pandemic (see Döring, 2020). Thus, the current study further proposed that sexual functioning might also serve as an adaptive mechanism in response to the COVID-19 pandemic, potentially helping to buffer individuals from adverse impact of COVID-19 related stress in partnered individuals with rich and rewarding sex lives. We posit that this offers individuals with an immediate and powerful method of managing stress, reprioritizing their relationship, and connecting with their romantic partner, thereby serving as a source of resilience. Consistent with this, sexual functioning has been linked to greater satisfaction with life, mental health, physical health, lower resting heart rate variability, and longevity (e.g., Costa \& Brody, 2012; Levin, 2007; Palmore, 1982).

Spillover from Sexual Functioning to Romantic Functioning. The current study posits that sexual functioning might be most proximally impacted by the pandemic (with the upheaval of the pandemic affecting physical intimacy most immediately). We propose that romantic functioning will instead be indirectly impacted by the COVID-19 related stress via its robust links with sexual functioning (i.e., relationship dynamics souring when the sex lives within relationships deteriorate; Fig. 1A). This is consistent with findings demonstrating that sexual satisfaction predicted change in 
Fig. 1 Conceptual and MSEM models tested. Note: Rectangles and rectangle speech bubbles refer to specific observed variables within the study whereas ovals refer to the broader construct being assessed (in panel A) or to latent variables estimated from the observed variables (panel B). The constructs within each class (i.e., the COVID-19 related stress constructs, the sexual Functioning constructs, and the romantic functioning constructs) were allowed to correlate with one another to focus the analyses on associations between classes. The resulting models were fully saturated, and therefore gave perfect fit

\section{(A) Family Systems Theory applied to Romantic Relationships}

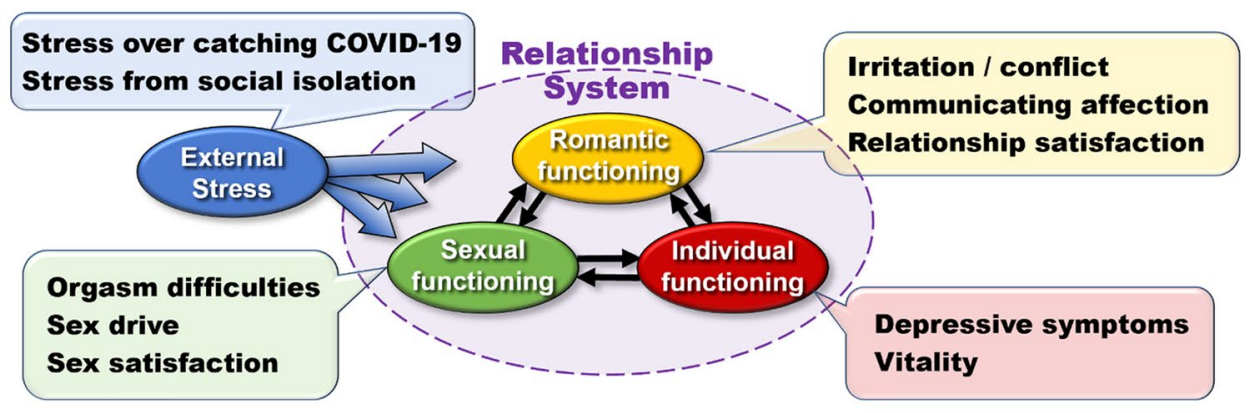

(B) Multilevel SEM model tested

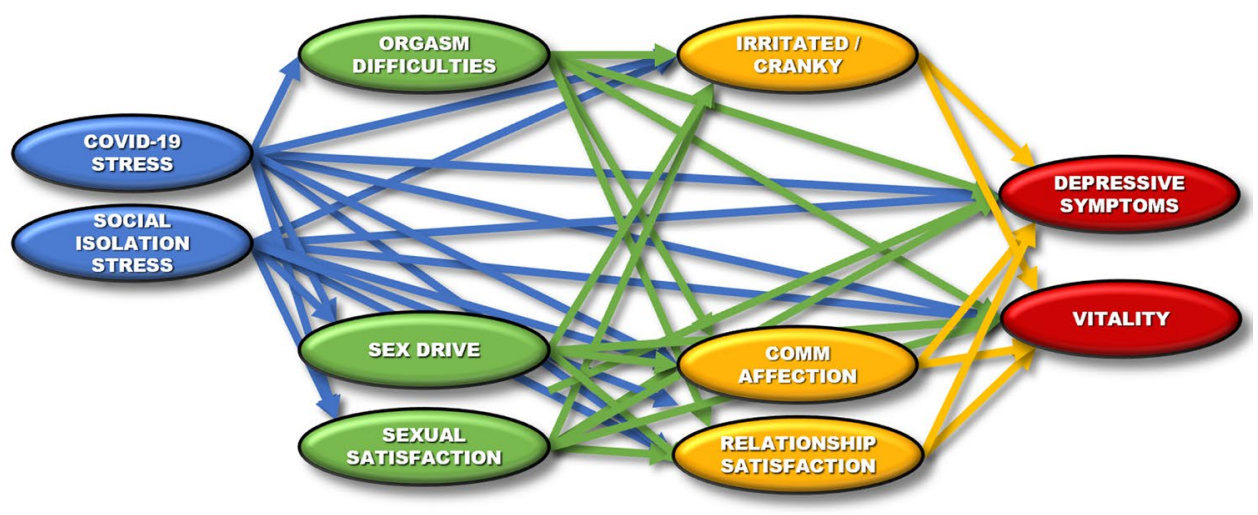

Level 2 -Stable between-person differences across the 6 months of the study

Level $1-$ Within-person change in each of the monthly waves of assessment

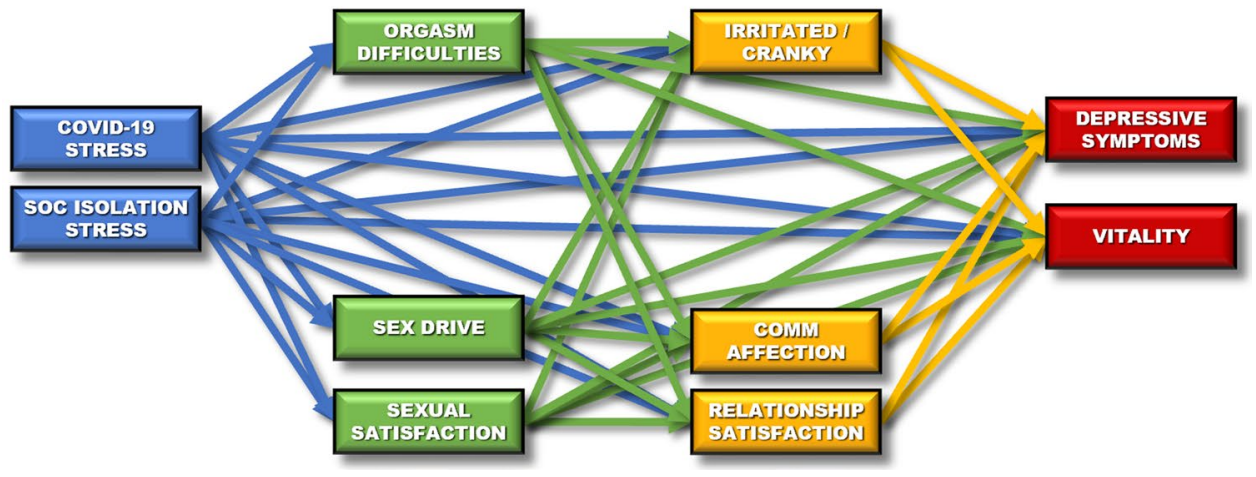

relationship satisfaction (Fallis et al., 2016). It is also consistent with findings linking orgasmic functioning to greater relationship quality (e.g., Brody \& Costa, 2009; Costa \& Brody, 2007; Hurlbert et al., 1993) and relationship investment (e.g., Ellsworth \& Bailey, 2013). Although the model tested in the current study therefore posits that disruptions of sexual functioning will lead to disruptions of broader romantic functioning, we recognize that the sexual and romantic systems within a relationship most likely exert reciprocal influence on one another across time. In fact, sexual and romantic satisfaction have demonstrated bidirectional associations over time in the early years of marriage (McNulty et al., 2016).
We therefore represent those links with bidirectional arrows in our conceptual model (Fig. 1A).

Romantic Dynamics as an Intermediate Mechanism. Sexual functioning has been directly linked to greater individual functioning and well-being (e.g., Chang et al., 2019; Sarti et al., 2010), and to lower levels of depressive symptoms (e.g., Buczak-Stec et al., 2021; Hollist et al., 2007). Despite this, the current model proposed that, for individuals within romantic relationships, romantic functioning might serve as a key intermediary process, tightly linked to both sexual functioning (e.g., McNulty et al., 2016) and individual functioning (e.g., Whitton \& Whisman, 2010) across time. Consistent 
with this, relationship satisfaction has been linked to lower depressive symptoms (e.g., Leach et al., 2013; Whitton \& Kuryluk, 2012) highlighting the importance of romantic relationships within individuals' lives. Although we are positing a specific direction of causality, it is likely that the sexual, romantic, and individual systems: (1) could each be directly impacted by stress associated with the COVID-19 pandemic, and (2) could also exert bidirectional or reciprocal influence on one another across time.

\section{The Current Study}

The current study sought to investigate a multi-stage, processoriented mediation model based on Family Systems Theory, (Broderick, 1993; Minuchin, 1985; see Fig. 1A). Specifically, our study sought to integrate: (1) $30+$ years of research on sexual health (by treating it as a multidimensional phenomenon), (2) $60+$ years of research on romantic relationships and marriage (by including irritability/conflict, affection, and relationship satisfaction as central processes arising out of that literature), (3) $60+$ years of clinical psychology research on psychological distress, (4) research highlighting increased distress during the pandemic, (5) $20+$ years of research on positive psychology (by including vitality as a separate outcome), and (6) the emergence of two distinct forms of COVID-19 related stress (i.e., stress over potentially contracting COVID-19 and the stress of social isolation secondary to lockdown policies). Thus, our model (Fig. 1) linked those COVID-19 related stressors to individual wellbeing (depressive symptoms and levels of energy/vitality) via sexual functioning (difficulties with orgasms, sex drive, and sexual satisfaction) and romantic functioning (irritability/ conflict with a partner, communicating affection, and relationship satisfaction) as key sets of interpersonal processes that could shape the impact of the pandemic on the lives of individuals. Toward that end, an online sample of 1241 participants in relationships completed a baseline survey followed by 6 monthly surveys during the early stages of the COVID-19 pandemic in the US.

Hypotheses. We hypothesized that external stressors due to the pandemic would impact all systems within the relationship (i.e., the blue levels throughout Figs. 1A and 1B; Hypothesis 1). We specifically hypothesized that greater levels of COVID-19 related stress would predict poorer sexual functioning (Hypothesis 1A), poorer romantic functioning (Hypothesis 1B), and poorer individual functioning (Hypothesis 1C). We further hypothesized that greater sexual functioning would predict greater romantic functioning (Hypothesis 2; green arrows in Fig. 1B). Finally, we hypothesized that greater romantic functioning would predict greater individual functioning (Hypothesis 3; yellow arrows in Fig. 1B). Consistent with previous findings supporting sexual and relationship functioning as mechanisms (e.g., Fallis et al., 2016;
Harper et al., 2000), we hypothesized that significant indirect paths would emerge from the proposed model suggesting that sexual functioning (Hypothesis 4A) and romantic well-being (Hypothesis 4B) would mediate the associations between COVID-19 related stressors and individual well-being across the 6 months of the study.

Exploring Potential Moderators. Given findings suggesting differences in sexual functioning across demographic groups like gender (e.g., Frederick et al., 2018; Laumann et al., 2000), sexual orientation (e.g., Garcia et al., 2014; Peplau \& Fingerhut, 2007), and age (e.g., Haavio-Mannila \& Kontula, 1997), the study also sought to evaluate the degree to which the results presented would generalize across distinct demographic groups. We therefore ran a series of multigroup models evaluating race, age, gender, sexual orientation, relationship stage, and cohabitation status as possible moderators.

\section{Method}

\section{Participants and Procedure}

All procedures and materials for this study were approved by an Institutional Review Board. Informed consent was obtained from all participants by providing a consent form as the first webpage of the survey. All participants were required to be at least 18 years old, and currently in a romantic relationship. Although not restricted to United States residents, recruitment was targeted to individuals living within the US and consequently, $98 \%$ of the respondents were from the US. The survey was presented online via SurveyGizmo.com, took approximately $30-35$ min to complete, and was advertised as "The Finding Pleasure in Sex Study." Participants were recruited from various online platforms: ResearchMatch (56\%), a psychology undergraduate participant pool (14\%), emails (11\%), Amazon.com's Mechanical Turk crowdsourcing service (10\%), Reddit forums (6\%; e.g., SampleSize), and other sources (3\%; e.g., Facebook, University of Hanover, the second author's lab website). These recruitment efforts yielded a sample of 1,241 individuals who were currently in romantic relationships. Individualized feedback served as the primary recruitment incentive. Participants recruited through MechanicalTurk also received $\$ 0.50$ of store credit for Amazon.com, and respondents from the undergraduate participant pool also received extra credit toward their psychology courses.

Monthly Follow-up Surveys. Participants were offered the option of providing an email address at the beginning of the baseline survey so that they could be invited to participate in 6 monthly follow-up surveys, and 1,033 (83\%) provided an email address. Those respondents were sent up to 3 email invitations for each of the 6 monthly follow-ups. 
Of those respondents, 624 (60\%) participated in at least one monthly follow-up, completing an average of 3.0 follow-ups $(S D=1.7)$. Analysis of variance (ANOVA) and chi-squared analyses contrasting respondents participating in the followups from those not providing follow-up data failed to uncover significant differences across income groups $\left(\chi^{2}(3)=4.5\right.$, $p=0.217)$ or baseline levels of vitality $(F(1,1044)=1.8$, $p=0.179)$. However, these analyses suggested that participants providing follow-up data were: slightly more likely to be women $\left(\chi^{2}(2)=9.2, p=0.010,64 \%\right.$ women among participants providing follow-up data vs. $61 \%$ women among participants not providing follow-up data), less likely to be heterosexual $\left(\chi^{2}(4)=9.9, p=0.043,55 \%\right.$ in participants with follow-up vs. $62 \%$ in participants without follow-up), more likely to have a graduate degree $\left(\chi^{2}(3)=29.8, p<0.001\right.$, $36 \%$ in participants with follow-up vs. $25 \%$ in participants without follow-up), more likely to be engaged/married $\left(\chi^{2}(2)=10.5, p=0.005,51 \%\right.$ in participants with follow-up vs. $43 \%$ in participants without follow-up), more likely to be white $\left(\chi^{2}(1)=11.1, p=0.001,85 \%\right.$ in participants with follow-up vs. $78 \%$ in participants without follow-up), and older $\left(F(1,1239)=53.3, p<0.001, \eta^{27}=0.041\right)$, with slightly fewer depressive symptoms $(F(1,1054)=16.9, p<0.001$, $\eta^{2}=0.016$ ). It should be noted that the sheer size of the current sample made it possible to detect even subtle attrition differences as statistically significant. However, when taken as a set, these analyses revealed generally mild attrition differences, supporting the use of the longitudinal data.

A total of 1,241 respondents completed the baseline survey from March 5th to May 5th, 2020. As seen in Table 1, the participants were predominantly women (62\%) and Caucasian (82\%), with 3.7\% Black/African American, 8.7\% Asian, $3.9 \%$ multiracial, and $1.7 \%$ other or unknown. When asked separately about ethnicity, $7.2 \%$ identified as Hispanic or Latinx. The average age was 35.2 years old, with an average income of $\$ 76,276(\mathrm{SD}=\$ 37,934)$. The sample represented a diversity of sexual orientations, with only $59 \%$ of the respondents identifying as heterosexual. The sample was also diverse in ages represented (from 18 to 80 years old), with a majority of the respondents in their $20 \mathrm{~s}$ and early $30 \mathrm{~s}(62 \%)$. The sample contained a range of income levels but was fairly well educated, with $77 \%$ reporting having earned a bachelor's or graduate degree. The sample also contained a range of relationship stages and lengths (from 0.1 to 56.2 years). However, a majority of the respondents were either married (47\%; together an average of 14.2 years, married an average of 12.5 years) or in long-term committed relationships (28\%; together an average of 5.2 years). Consistent with that, $61 \%$ of respondents were living with their romantic partners for an average of 17.2 years $(S D=131.7)$, with another $26 \%$ reporting living separately but spending some or most nights together, and 9\% currently in long-distance relationships.

\section{Measures}

Recruitment and the collection of baseline survey data began in early March 2020, just before the COVID-19 pandemic swept across the US. As a result, the baseline survey did not contain any assessments of COVID-19 related stress. However, just 2 weeks after the start of recruitment (in mid-March 2020 - as the pandemic began to draw national attention within the US and lockdown procedures were being implemented), the COVID-19 related constructs were quickly added to the monthly follow-up surveys. The assessments of the remaining constructs were identical across all 7 waves of assessment (baseline and all 6 monthly followups) as detailed below. As the follow-up surveys were spaced 1 month apart and to ensure that we would be able to capture behavior that might happen less frequently, a majority of the scales instructed respondents to think of the last month when selecting their responses. The two primary exceptions to this were the Patient Health Questionnaire (PHQ-9) and our measure of vitality which instructed respondents to think of the last 2 weeks. This allowed us to retain the originally validated time frame for those scales, allowing them to remain grounded in a broad clinical literature measuring symptoms and individual functioning.

COVID-19 Stress Respondents completed 4 items (developed for the current study based on preliminary data from another COVID-19 focused study; see Daks et al., 2020) reporting on their experience of stress related to health concerns during the pandemic. The items were presented with a stem question ("In the last month, how much were you worried or stressed about each of the following...") and assessed for a variety of health concerns (i.e., "the ongoing possibility of exposure to COVID-19," "your own health," "the health of loved ones," "news reports on the crisis"). The items were rated on a 6-point scale ("Not at all or NA" to "Extremely"), and responses to these items were averaged so that higher scores indicated higher levels of COVID-19 stress $(\alpha=0.80)$.

Social Isolation Stress Respondents completed 3 items (developed for the current study based on preliminary data from another COVID-19 focused study; see Daks et al., 2020) assessing stress (using the stem "In the last month, how much were you worried or stressed about each of the following...") from social isolation during the pandemic (using the items "social isolation," "boredom," "cabin fever / going stir crazy"). These items were rated on a 6-point scale ("Not at all or NA" to "Extremely") and responses were averaged so that higher scores indicated higher levels of social isolation stress $(\alpha=0.84)$.

Orgasm Difficulties Respondents were asked to report on their general difficulty achieving orgasms with the item: "In general, how difficult is it for you to reach orgasm (climax) during sexual activity?" This item was developed for the current study (based on typical item content for this construct 


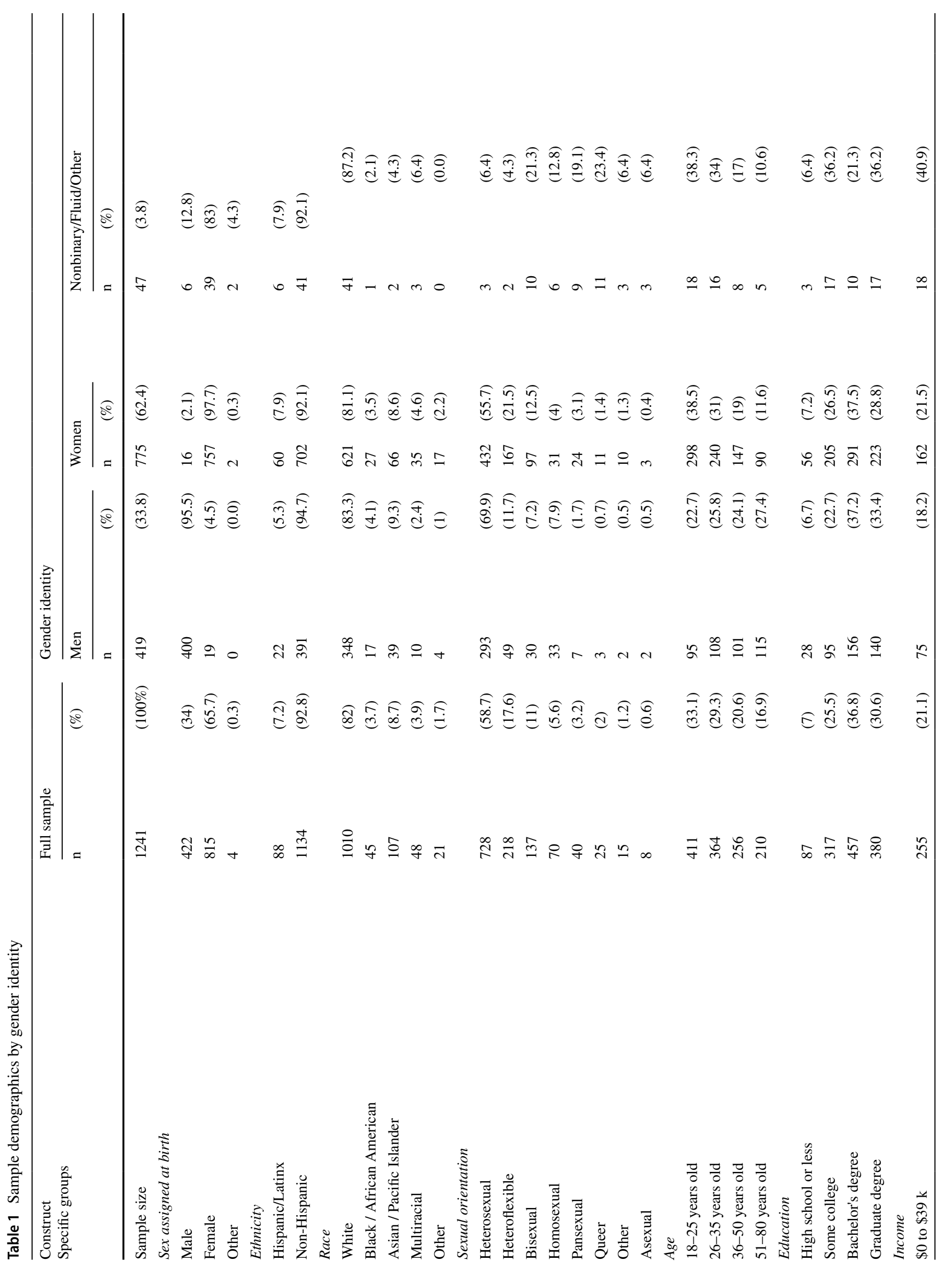




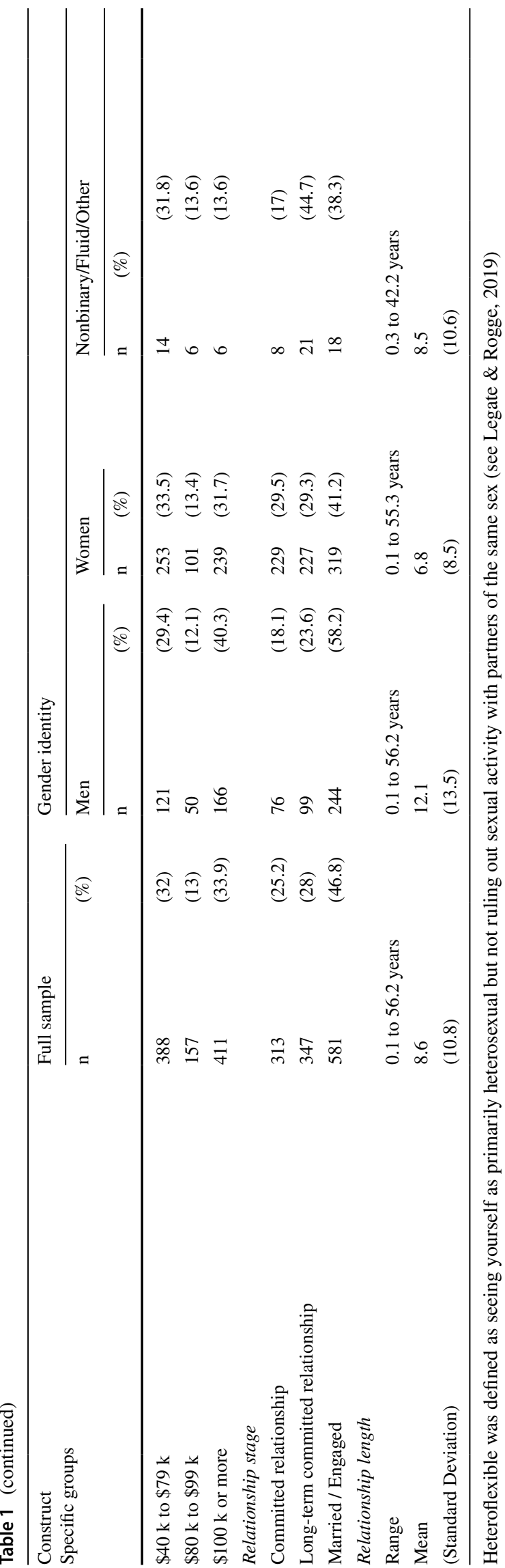

from the larger literature) and was rated on a 6-point scale ("Not at all" to "Extremely"), with higher scores indicating greater difficulty achieving orgasms.

Sex Drive Respondents completed 4 items developed for the current study which were prototypical of the content used to assess sex drive in previous work (e.g., Baumeister et al., 2001). The items ("I had a strong sex drive," "I frequently thought about sex," "It didn't take much to get me sexually excited," "I thought about sex almost every day") were presented with a stem ("In the last month..."), and were rated on a 6-point scale ("Not at all" to "Extremely"). Responses to these items were averaged so that higher scores indicated higher levels of sex drive $(\alpha=0.90)$.

Sexual Satisfaction Respondents completed the 6-item Quality of Sex Index (QSI-6; Shaw \& Rogge, 2016) assessing sexual satisfaction (e.g., "my sex life was fulfilling"). The items were presented with a stem ("Thinking of the last month..."), and were rated on a 6-point scale ("Not at all true" to "Completely true"). Responses to these items were totaled using the validated scoring system so that higher scores indicated higher levels of sexual satisfaction $(\alpha=0.98)$.

Irritation with Partner Respondents completed 4 items reporting on their experience of irritation with their partner. The items ("My partner ended up stressing me out," "My partner and I started getting on each other's nerves," "My partner was a source of irritation," "My partner and I got a bit cranky with each other") were presented with the stem question, "In the last month, how much were each of the following true?" These items were designed for the current study in an effort to capture low grade levels of negative conflict and irritability that might arise within romantic relationships in the context of a global pandemic, and were rated on a 6-point scale ("Not at all" to "Extremely"). Responses to these items were averaged so that higher scores indicated higher levels of irritation with partner $(\alpha=0.93)$.

Communication of Affection Respondents completed 5 items assessing communication of affection. To focus on communicating affection as a dynamic process, five items with more state-like properties were developed based on the content of the Trait Affection Given scale (TAG; Floyd, 2002): "I was always telling my loved ones how much I care about them," "when I felt affection for someone, I usually expressed it," "I was pretty good at telling the people I care about know much they mean to me," "I never missed an opportunity to tell loved ones know how important they are to me," and "I often told people how much I love them." The items were presented with the stem, "In the last month...", and were rated on 6-point scales ("Strongly disagree" to "Strongly agree"). Responses to these items were averaged so that higher scores indicated higher levels of communication of affection $(\alpha=0.94)$. 
Relationship Satisfaction Respondents completed the 4-item Couples Satisfaction Index (CSI-8; Funk \& Rogge, 2007) assessing relationship satisfaction (e.g., "I had a warm and comfortable relationship with my partner"). The items were presented with the stem, "Thinking of the last month...", and were rated on 6 and 7-point scales (e.g., "Not at all" to "Completely"). Responses to these items were totaled using the validated scoring system so that higher scores indicated higher levels of relationship satisfaction $(\alpha=0.95)$.

Depressive Symptoms Respondents completed the 9-item Patient Health Questionnaire (PHQ-9; Spitzer et al., 1999) assessing depressive symptoms. The items (e.g. "feeling down, depressed, or hopeless") were presented with the stem, "Over the last two weeks, how often have you been bothered by the following problems?", and were rated on a 4-point scale ("Not at all," "Several days," "More than half the days," "Nearly every day"). Responses to items were totaled using the validated scoring system so that higher scores indicated higher levels of depressive symptoms $(\alpha=0.90)$.

Vitality Respondents completed 6 items assessing their vitality and energy. The items ("I had energy and spirit," "I felt energized," "I felt alive and vital," "I had a lot of energy," "I felt really "up" or lively," "I felt full of pep") were presented with the stem, "In the last two weeks..." and were rated on a 6-point scale ("Not at all" to "Extremely"). These items were identified from a larger pool of vitality and positive affect items that were: (1) drawn from existing measures such as the Subjective Vitality Scale (Ryan \& Frederick, 1997) and the Mood and Anxiety Symptom Questionnaire (Watson et al., 1995), (2) given to over 3,000 online respondents, and (3) identified as measuring a single common construct using Exploratory Factor Analyses, and (4) identified as the most informative and effective items for assessing vitality using Item Response Theory analyses run by the second author. Responses to items were averaged so that higher scores indicated higher levels of vitality $(\alpha=0.97)$.

\section{Data Analysis}

The proposed mediation model (Fig. 1B) was tested within a multilevel SEM framework following current recommendations (Preacher et al., 2010), in which repeated observations across time at level 1 were nested within individual participants at level 2. Thus, stable levels of each construct across the 6 months of the study were modeled as latent variables (presented as ovals) at level 2, representing stable betweenperson differences (essentially individual average levels of each construct across the 6 months of the study; the top half of Fig. 1B). In contrast, shifts in each construct within specific waves of assessment were modeled as observed variables (presented as rectangles) at level 1, representing withinperson change across time (effectively shifts above and below each individual's own average levels of each construct within specific months of assessment; the bottom half of Fig. 1B). This allowed the associations predicted by the model to be tested both at the between-person level (associations between stable and more chronic levels of the constructs spanning the full 6 months of the study) and at the within-person level (examining associations among residual shifts in the various constructs within specific waves of assessment, focusing on more acute links). Although not shown in Fig. 1B, each construct was allowed to correlate with the other constructs within its own class (e.g., sexual functioning variables correlating with one another, romantic functioning variables correlating with one another), focusing the analyses on the predictive associations between classes of constructs. In addition, dichotomous variables coding parent status and employment status were included as level 2 controls predicting all other variables to control for their possible effects. The final model was fully saturated and therefore yielded perfect fit. As standardized path coefficients are not available in multilevel modeling, the variables were standardized at level 1 (i.e., across all participants and across all waves) prior to running the analyses to obtain rough approximations of standardized path coefficients. As bootstrapping is not available within multilevel modeling in Mplus, indirect paths were tested using the Rmediation online package (Tofighi \& MacKinnon, 2011) to estimate their asymmetric confidence intervals. Given the size of our sample and the sheer number of paths estimated, we focused our results narrative on the paths that were (1) significant at $p<0.01$ and (2) had path coefficients with absolute values $\geq 0.10$. This helped to ensure that the paths being interpreted accounted for greater amounts of predictive variance and were more likely to replicate in future samples. The model was run in Mplus 7.2 using full information maximum likelihood (FIML) estimation to handle missing data.

\section{Results}

\section{Characteristics of the Sample}

Stress and Individual Functioning in the Sample As seen in Table 2, on average respondents reported feeling somewhat worried/stressed about their own health, the health of loved ones, and the risk of contracting COVID-19 along with moderate levels of stress from social isolation across the 6 months of the study. Consistent with this, although the respondents typically reported minimal to mild levels of depressive symptoms (73\% minimal, $14 \%$ mild), $7 \%$ of the PHQ-9 scores at baseline suggested moderate depressive symptoms and $6 \%$ suggested severe, indicating that the study captured a range of psychological distress occurring at the start of the pandemic. Correspondingly, on average respondents reported only moderate levels of vitality, typically only feeling only somewhat 
energized and vital each day during the early stages of the pandemic.

Sexual and Romantic Functioning in the Sample. On average, respondents also reported fairly moderate levels of sexual functioning, typically reporting feeling only somewhat satisfied with their sex lives with their partners, experiencing mild to moderate difficulties with orgasms, and moderate sex drives across the first 6 months of the pandemic in the US. Consistent with previous findings suggesting consistent gender differences on achieving orgasms (e.g., Frederick et al., 2018; Laumann et al., 2000) and sex drive (e.g., Baumeister et al., 2001) but failing to identify gender differences on sexual satisfaction (e.g., Shaw \& Rogge, 2016), women in the current sample reported greater difficulties with orgasms across time than men $\left(M_{\text {women }}=2.8, M_{\text {men }}=2.1\right.$, $d=0.47, t(2978)=12.1, p<0.001)$ and lower sex drives across time than men $\left(M_{\text {women }}=3.0, M_{\text {men }}=4.0, d=-0.72\right.$, $t(2747)=-17.9, p<0.001)$, but failed to demonstrate significant differences from men on sexual satisfaction across time $\left(M_{\text {women }}=17.5, M_{\text {men }}=16.9, d=0.06, t(2696)=1.6\right.$, $n s$ ). Turning to the quality of their romantic relationships, on average respondents were fairly happy in their relationships, with satisfaction scores on the upper range of that measure. However, there was also quite a bit of variability in relationship satisfaction, with nearly a quarter of the respondents (24\%) experiencing meaningful levels of relationship dissatisfaction (i.e., CSI-4 scores falling below the established cut score; see Funk \& Rogge, 2007). Consistent with this, on average respondents reported low to moderate levels of irritability within their relationships across the first 6 months of the pandemic, typically getting a little to somewhat cranky and irritated with one another and doing a fairly good job of telling their loved ones how much they cared. Taken together, these results suggest that the current study captured a diverse sample of US adults in reasonably long-term relationships, who varied in their levels of sexual, romantic, and individual functioning across the first 6 months of the COVID-19 pandemic. As seen in the bivariate correlations presented in Table 2, the various constructs examined in the current study demonstrated low to moderate associations with one another in the expected directions across time, suggesting appropriate levels of collinearity to support the planned multivariate analyses.

\section{Multilevel SEM Results}

Structure of the Model Following current guidelines (Preacher et al., 2010), we evaluated the proposed mediational model within a multilevel framework: (1) at the level of stable between-person differences across the 6 months of the study (i.e., level 2 latent variables representing average levels of each construct for each individual across the study), and (2) at the level of within-person fluctuations in the constructs within specific waves of assessment (i.e., level 1 observed variables representing fluctuations above and below each individual's own average levels of each construct within specific months). This not only allowed us to appropriately model the nested nature of our data (i.e., repeated observations within individuals), but also allowed us to distinguish those two sources of predictive associations. As parent status and employment status could have affected multiple systems within relationships during the pandemic, we included those as controls at level 2 in all models tested to help ensure that the results observed were not driven by those demographic variables.

Establishing Generalizability of the Findings To ensure that the results presented would generalize across distinct demographic groups, we first ran a series of multigroup models evaluating race, age, gender, sexual orientation, relationship stage, and cohabitation status as possible moderators. Within each of these analyses, we would run the model in a set of demographic groups, constraining the paths in the model to be identical across the groups. A significant lack of fit in one of these models would have suggested that the results meaningfully differed across that set of demographic groups, thereby identifying a significant moderator. However, as shown in Table 3, these multigroup models demonstrated excellent levels of fit when constrained across the various demographic groups tested, failing to identify significant moderation and suggesting that the results of the model generalized across those groups. These findings specifically suggest that race, age, gender, sexual orientation, relationship stage, and cohabitation status did not serve as confounding variables underlying the current results. Given these findings, the remainder of the results focused on a model estimated across the entire sample. As that model was fully saturated, it gave perfect fit.

Level 2 Prediction Linking Stable Levels of the Constructs across 6 Months Table 4 presents the path coefficients examining the proposed mediation model at level 2, and Figs. 2A, 2B, and 2C present the most robust paths emerging from that level of the model (whereas Table 5 presents the level 1 findings). The latent variables created at this level (represented by ovals in the figures) were created from the repeated observations of each construct (modeled at level 1 and represented by squares as they represent observed variables). As a result, those level 2 latent variables represent the stable levels of each construct for each participant across the entire 6 months of the study (i.e., the shared variance across all of those observations, effectively each participant's average on that measure across all waves). As seen in Fig. 2B, higher average levels of stress due to social isolation during the first 6 months of the pandemic were linked to greater average irritability with romantic partners, greater average depressive symptoms, and lower average levels of vitality across those 6 months. Similarly, higher average levels of 


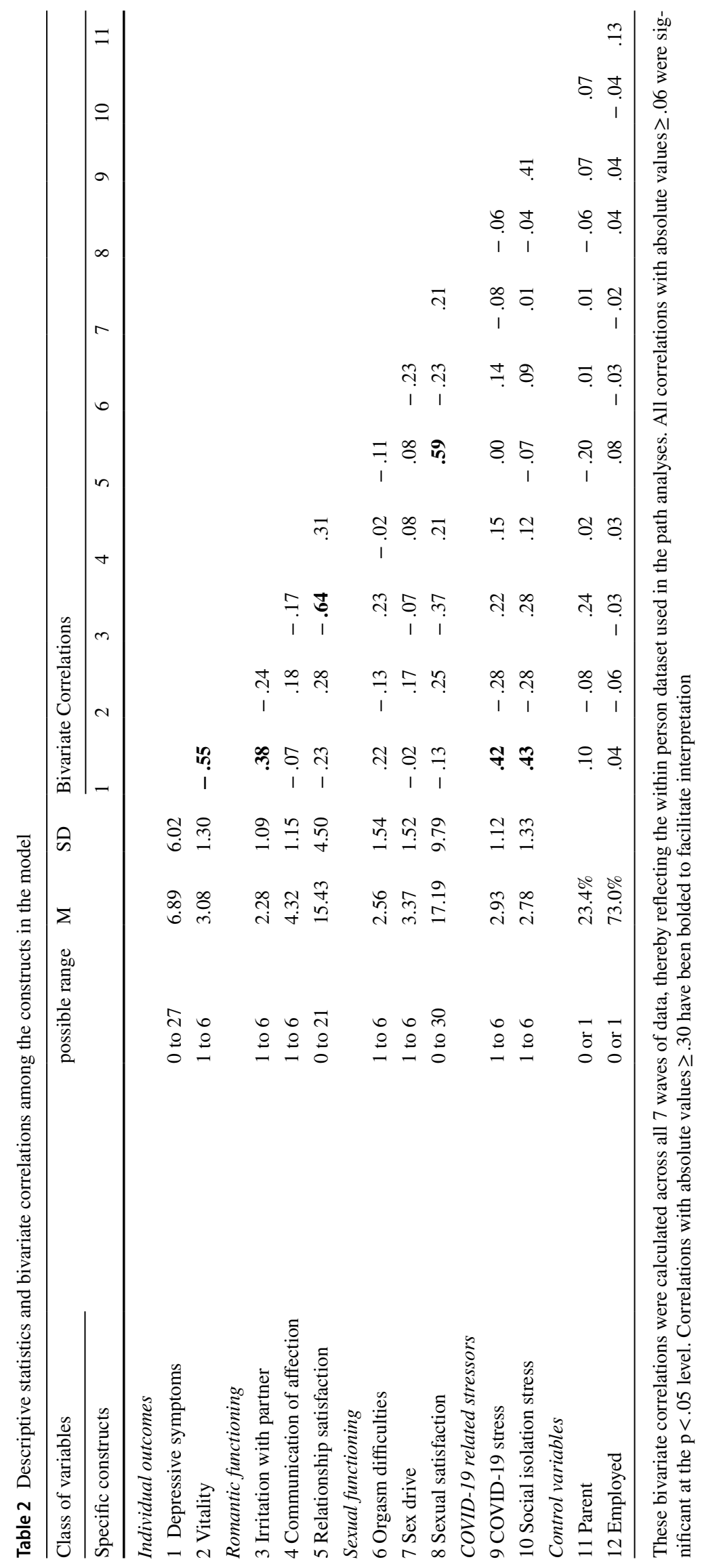


Table 3 Fit statistics for multigroup models evaluating possible moderators of the model by constraining the effects to be identical across groups

\begin{tabular}{|c|c|c|c|c|c|c|c|c|}
\hline \multirow{2}{*}{$\begin{array}{l}\text { Groups being compared in constrained } \\
\text { multigroup analyses }\end{array}$} & \multirow[t]{2}{*}{ Free Param } & \multicolumn{3}{|l|}{$\chi^{2}$} & \multirow[t]{2}{*}{ RMSEA } & \multirow[t]{2}{*}{ CFI } & \multicolumn{2}{|l|}{ SRMR } \\
\hline & & Est & $\mathrm{df}$ & $\mathrm{p}$ & & & Within & Between \\
\hline White vs. Non-White & 200 & 146.85 & 90 & .0001 & .016 & .984 & .079 & .050 \\
\hline $18-24$ yo vs. $25-35$ yo vs. $36-80$ yo & 255 & 214.671 & 180 & .0395 & .011 & .990 & .042 & .067 \\
\hline Identifying as Men vs. Women & 200 & 145.927 & 90 & .0002 & .016 & .984 & .032 & .049 \\
\hline Heterosexual vs. Non-Heterosexual & 200 & 138.921 & 90 & .0007 & .015 & .986 & .025 & .052 \\
\hline $\begin{array}{l}\text { Committed vs. Long-term Committed } \\
\text { vs. Engaged/Married }\end{array}$ & 255 & 235.701 & 180 & .0033 & .014 & .985 & .043 & .059 \\
\hline Living together vs. Living apart & 200 & 114.498 & 90 & .0417 & .011 & .993 & .030 & .047 \\
\hline
\end{tabular}

These models were run in Mplus 7.11 and constrained the paths between constructs in the multilevel model to be identical across groups. The fit indices for the unconstrained multigroup models are not shown as those models were fully saturated and therefore gave perfect fit. yo =years old

COVID-19 related stress were linked to greater average difficulties with orgasms, greater average depressive symptoms, and lower average levels of vitality. Taken together, these results support Hypothesis 1 and highlight challenges to sexual, romantic and individual functioning across the first 6 months of the pandemic. Unexpectedly, higher average levels of COVID-19 stress across the 6 months of the study were also linked to greater average levels of communicating affection to loved ones across that time period, suggesting that the risk of contracting COVID-19 or losing a loved one might have helped individuals to reprioritize their close relationships and reach out to those they love.

Indirect Paths at Level 2 As show/n in Fig. 2C and the top half of Table 6, a number of significant indirect paths emerged supporting the proposed model, and more specifically Hypotheses 2, 3, and 4. Thus, average levels of COVID19 related health stress across the 6 months of the study were indirectly linked to greater average irritation with a romantic partner and greater average depressive symptoms via the higher average levels of difficulties with orgasms they predicted across the study (supporting Hypothesis 4). In contrast, the direct links between average levels of COVID-19 stress across the 6 months and poorer average individual functioning across that time interval were significantly weakened by average levels of COVID-19 stress predicting greater average levels of communicating affection to loved ones, which in turn predicted lower average depressive symptoms and higher average levels of vitality. Finally, the results suggested that average levels of sexual satisfaction might have served as a source of resilience during the 6 months of the study, as they predicted greater average relationship satisfaction and greater average communication of affection (supporting Hypothesis 2), which in turn predicted greater average vitality and fewer average depressive symptoms (supporting Hypothesis 3), even after controlling for the impact of average levels of COVID-19 related stressors across the study.
Level 1 Prediction Linking Within-Person Change in the Constructs Table 5 presents the path coefficients examining the proposed mediation model at level 1, and Figs. 2D, $2 \mathrm{E}$, and $2 \mathrm{~F}$ present the most robust paths emerging from that level of the model. As seen in Fig. 2E, shifts above individuals' own average levels of social isolation stress (resulting from COVID-19 social isolation measures) within specific months of assessment predicted corresponding elevations in irritability with a romantic partner and depressive symptoms, and corresponding drops in sexual satisfaction and vitality within those same months. Thus, shifts in that form of COVID-19 related stress from month to month were linked to corresponding shifts in sexual, romantic, and individual functioning in those same months, offering support for Hypothesis 1.

Indirect Paths at Level 1 As shown in Fig. 2F and the bottom portion of Table 6 , a number of significant indirect paths emerged at level 1 supporting the proposed model and Hypothesis 4. Thus, elevations in social isolation stress above individuals' own average levels within specific months of assessment predicted corresponding individual elevations in irritation with partners in those same months by predicting corresponding drops in sexual satisfaction (Hypothesis 1), which in turn predicted stronger elevations in irritation with partners (Hypothesis 2). Similarly, the direct link between elevations in social isolation stress within specific months and corresponding drops in vitality in those same months was intensified by predicting corresponding drops in sexual satisfaction (Hypothesis 2), which in turn predicted further drops in vitality in those same months. Finally, two indirect paths emerged supporting both shifts in sexual functioning and shifts in romantic functioning as sequential mediators of the links between elevations in social isolation stress, and corresponding elevations in depressive symptoms and drops in vitality within specific months. Thus, elevations in social isolation stress within specific months predicted corresponding 


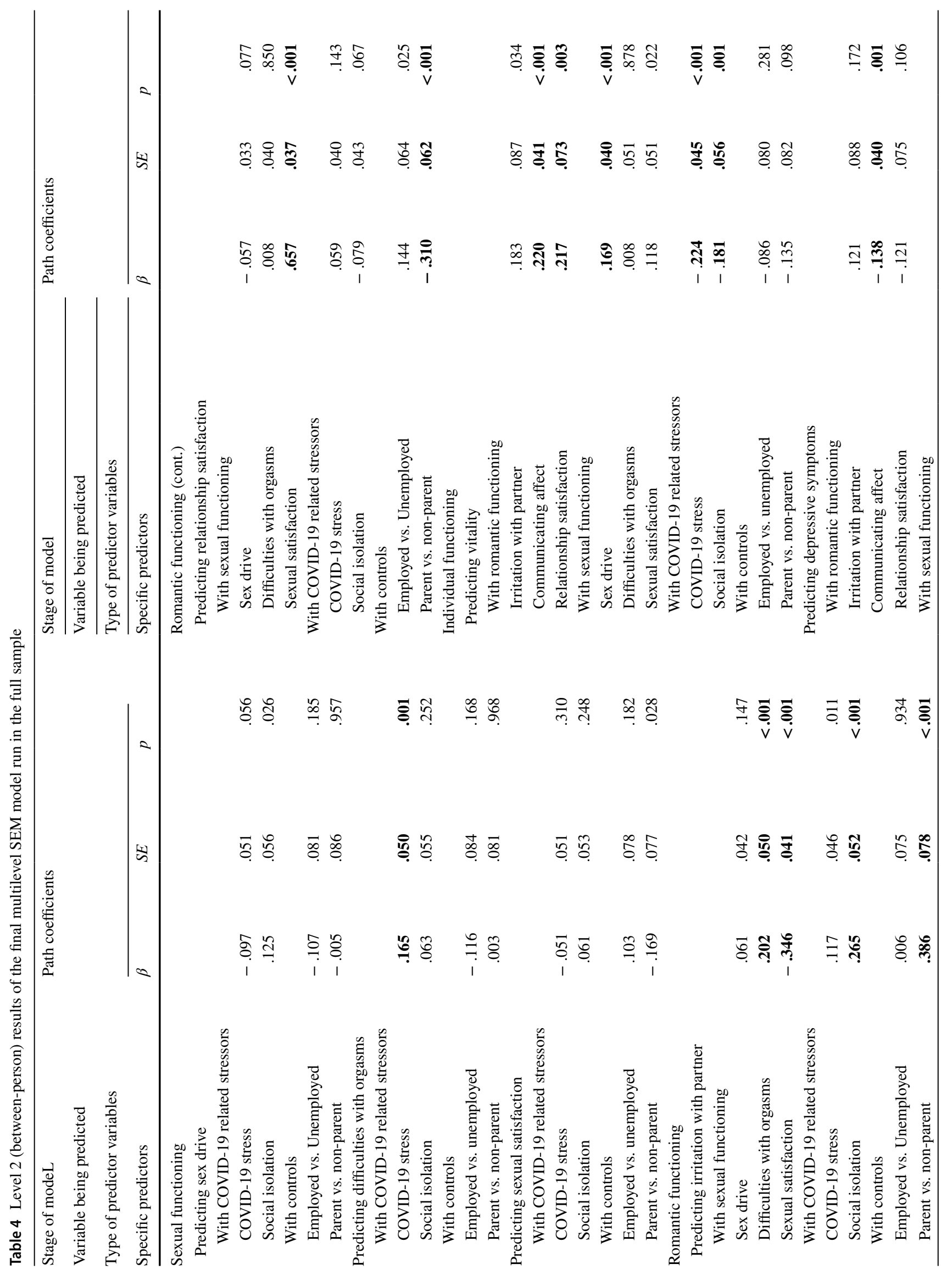




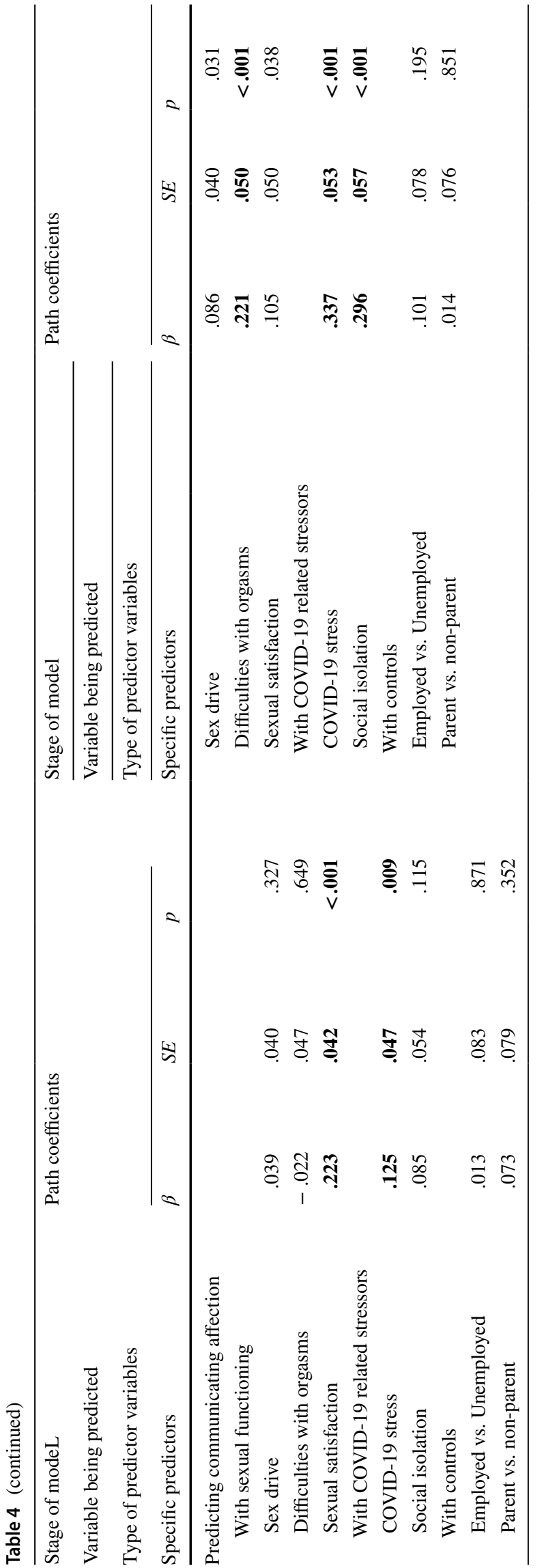

drops in sexual satisfaction (Hypothesis 1), which in turn predicted drops in relationship satisfaction (Hypothesis 2), which then predicted corresponding elevations in depressive symptoms and drops in vitality (Hypothesis 3 ) in those same months.

Although the model demonstrated invariance across gender of respondent (failing to identify gender as a significant confound), we ran secondary analyses including gender as an additional level 2 control. This allowed us to rule out the possibility that some of our results might have been driven by simple gender differences. Consistent with the model invariance across gender, the paths presented in Tables 4 and 5 and in Fig. 2 remained essentially unchanged, even after controlling for gender of respondent. Thus, the secondary analysis suggested that the current results were not only invariant across men and women but remained consistent even after controlling for mild gender differences.

\section{Discussion}

The current study sought to integrate key findings from the fields of individual psychopathology, research on sexual health, marital and couples research, and positive psychology by drawing key indicators from each of those fields into a large and comprehensive model. The primary goal of the study was therefore to draw upon those rich empirical traditions to develop a more nuanced understanding of precisely how the pandemic might have impacted sexual health, relationship health, and individual distress and well-being. The study applied Family Systems Theory (Broderick, 1993; Minuchin, 1985) as a conceptual framework to enrich our understanding of how the COVID-19 pandemic and the associated public health measures employed to flatten the curve (e.g., lockdowns, school and business closures, social distancing measures) might have impacted the lives of individuals in the US. Multilevel analyses in multi-wave data collected from a large online sample during the first 7-8 months of the pandemic in the US supported the proposed mediation at the level of within-person change across time. Thus, elevations in COVID-19 stress within specific months predicted corresponding drops in sexual functioning, which in turn predicted corresponding drops in romantic functioning, which in turn predicted corresponding drops in individual well-being. These findings highlight how the individual, sexual, and relationship systems are interconnected across time, such that disruptions in one system from an external stress (like a global health crisis) will likely spill over into other systems. In contrast, at the level of between-person differences, stable levels of sexual and relationship satisfaction across the 6 months of the study were unassociated with stable levels of COVID-19 stressors, representing sources of resilience that promoted well-being across the early stages of 
(A) All paths linking stable between-person differences

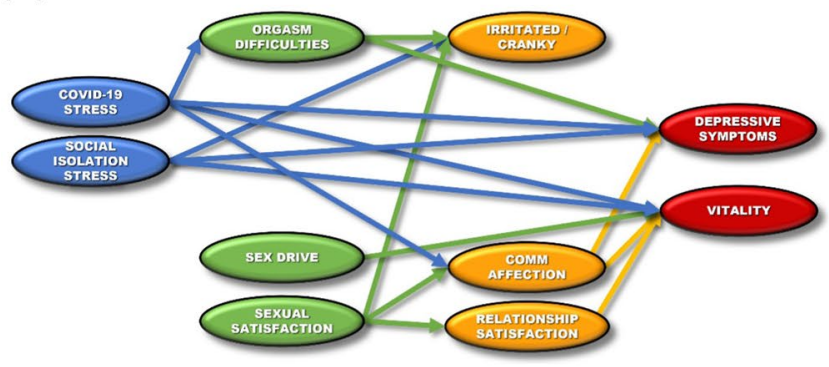

(B) Between-P predictive paths for COVID-related stressors

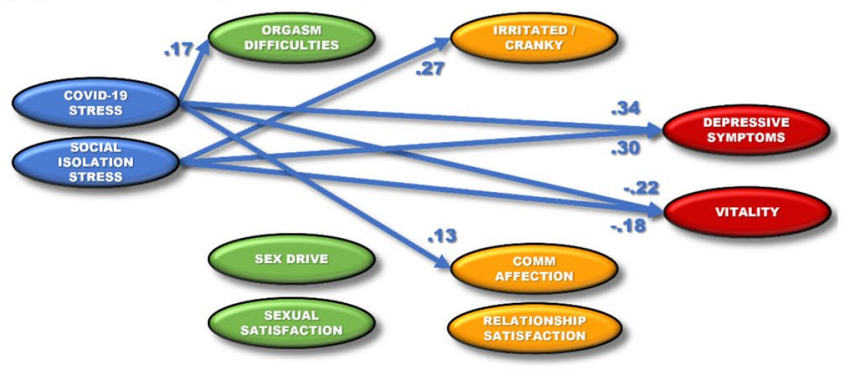

(C) Between-P indirect predictive paths

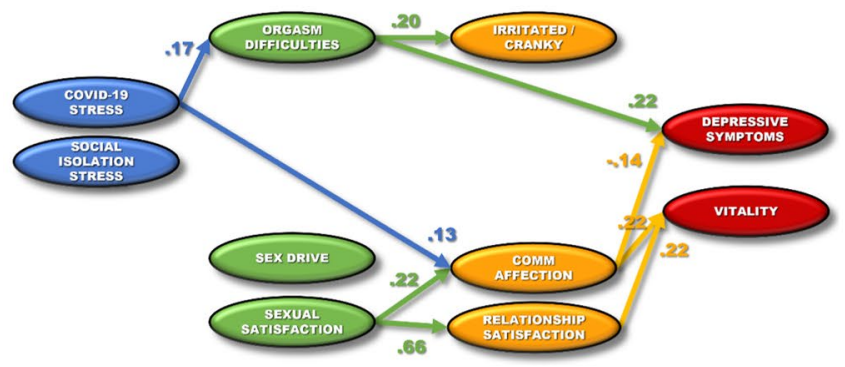

Fig. 2 Results of the MSEM mediation models. Note: Rectangles refer to specific monthly assessments of each variable within the study (level 1) whereas ovals refer to latent variables representing participants' average levels of each variable across the study (level 2).

the COVID-19 pandemic in the US. Extending those positive results, stable levels of stress from social isolation predicted stably higher amounts of communicating affection to one's loved ones, suggesting that individuals tend to reach out to those they love and strengthen those connections in the face of a massive world-wide crisis. Finally, stable difficulties with orgasms were linked to stably higher levels of irritability with partners and depressive symptoms, representing a key risk factor. Multigroup analyses suggested that the findings generalized across gender, age, race/ethnicity, sexual orientation, relationship stage, and cohabitation groups. Taken as a set, these findings underscored the critical nature of sexual and romantic functioning in the lives of individuals, highlighting potential sources of resilience/risk at the level of stable between-subject differences (e.g., stable levels of sexual and relationship satisfaction, and stable difficulties with orgasms), and potential warning signs and treatment targets on a monthly basis (e.g., sudden spikes in the stress
(D) All paths linking change within individuals over time

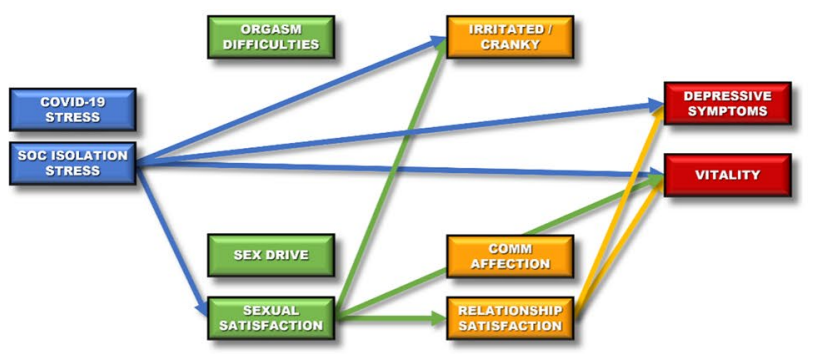

(E) Within-P predictive paths for COVID-related stressors

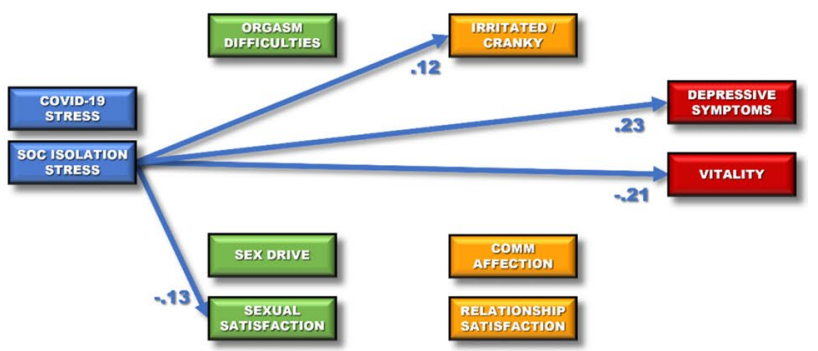

(F) Within-P indirect predictive paths

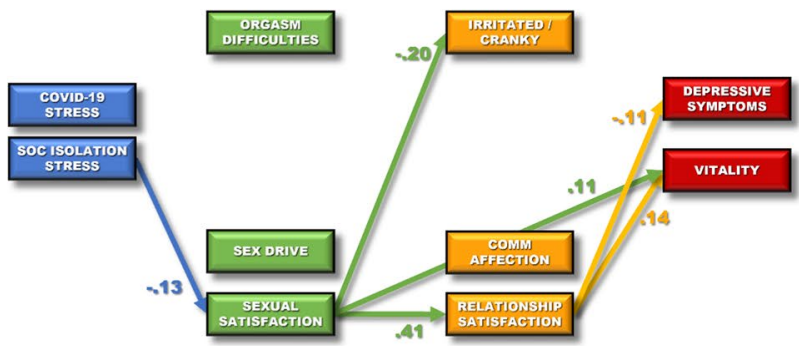

Only paths significant at $\leq .01$ and with absolute estimates $\geq .10$ are shown, focusing on the robust effects most likely to replicate in future work and accounting for more predictive variance

of social isolation, sudden drops in current sexual and/or relationship functioning). Thus, these findings offer relevant insights for clinicians and therapists working with individuals and couples during periods of acute stress like the global health crisis brought on by the COVID-19 pandemic.

\section{Implications}

Family Systems Theory Offers an Effective Conceptual Framework The current study represents an early attempt to apply the broader family systems framework to romantic relationships which may or may not include children. As the romantic dyad is already recognized as a key system within the larger family unit, we would posit that this application of family systems theory is simply focusing in on aspects of that theory that are relevant to childfree family units, as well as to romantic dyads before they start having children. Even without a child in the home, romantic couples can be 


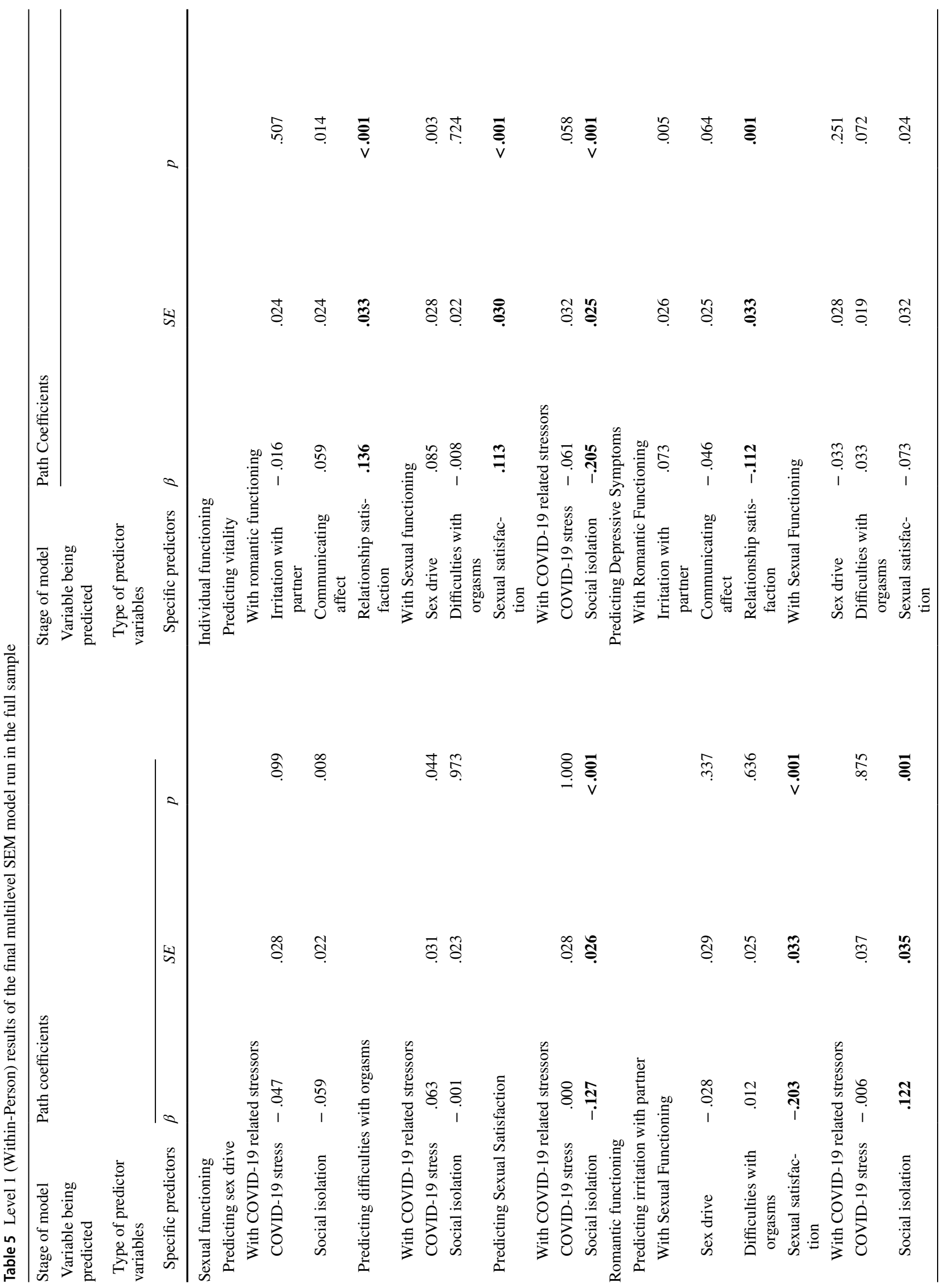




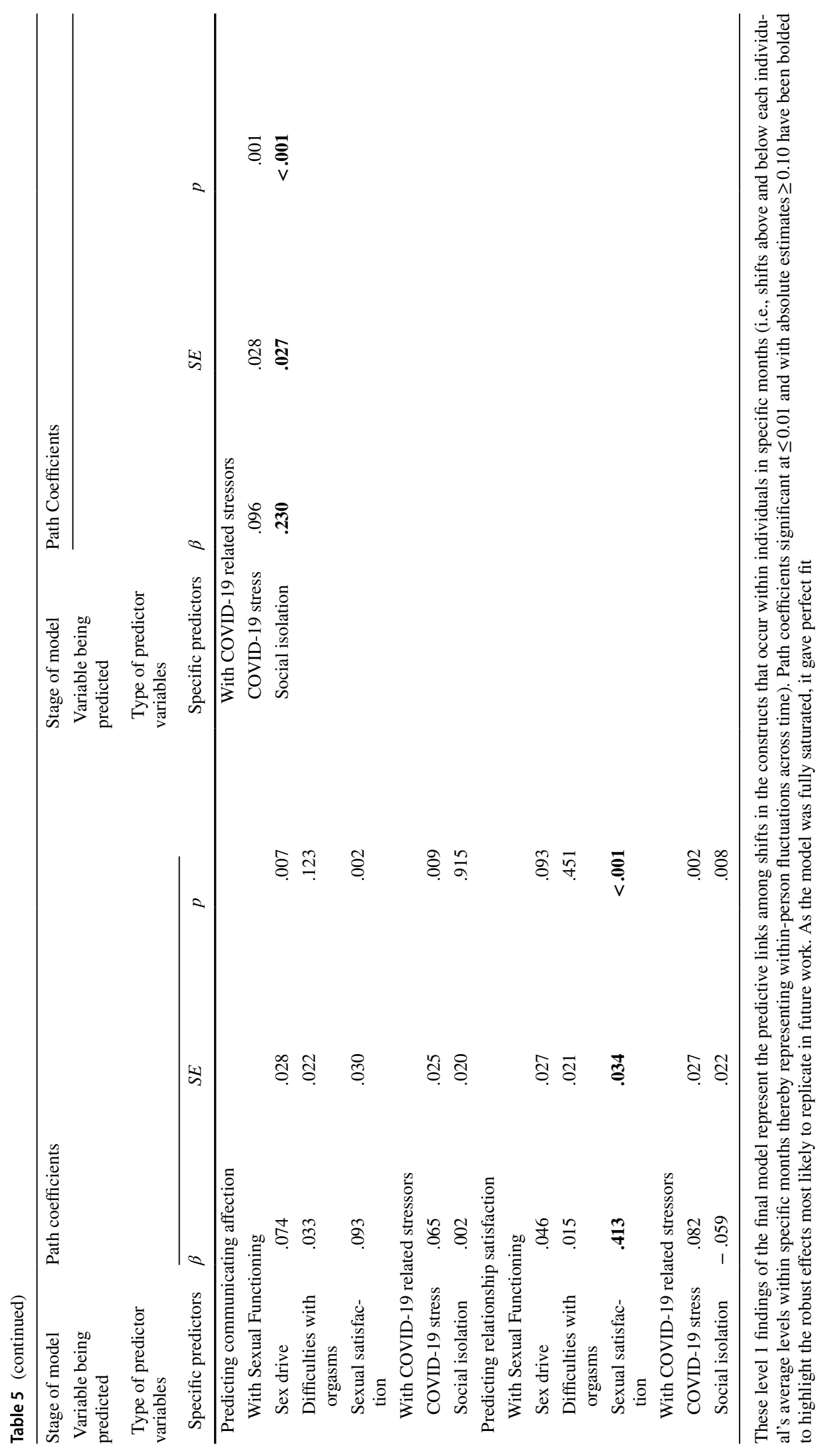


Table 6 Indirect paths suggested by the results

\begin{tabular}{|c|c|c|c|c|c|c|c|c|}
\hline \multirow{2}{*}{$\begin{array}{l}\text { Level of the model } \\
\text { Predictor }\end{array}$} & \multirow[b]{2}{*}{ Mediator } & \multirow{2}{*}{\multicolumn{2}{|c|}{ Outcome or Mediator 2}} & \multirow{3}{*}{\multicolumn{2}{|c|}{ Outcome }} & \multirow[b]{2}{*}{ est } & \multicolumn{2}{|c|}{$95 \% \mathrm{CI}$} \\
\hline & & & & & & & LL & UL \\
\hline \multicolumn{7}{|c|}{ Indirect associations among stable, between-person differences } & & \\
\hline COVID-19 stress & $\rightarrow$ Orgasm difficulties & $\rightarrow$ & Irritation with partner & & & .033 & .011 & .063 \\
\hline COVID-19 stress & $\rightarrow$ Orgasm difficulties & $\rightarrow$ & Depressive symptoms & & & .036 & .013 & .067 \\
\hline COVID-19 stress & $\rightarrow$ Communication of affection & $\rightarrow$ & Depressive symptoms & & & -.017 & -.036 & -.003 \\
\hline COVID-19 stress & $\rightarrow$ Communication of affection & $\rightarrow$ & Vitality & & & .028 & .007 & .053 \\
\hline Sexual satisfaction & $\rightarrow$ Communication of affection & $\rightarrow$ & Depressive symptoms & & & -.031 & -.054 & -.012 \\
\hline Sexual satisfaction & $\rightarrow$ Communication of affection & $\rightarrow$ & Vitality & & & .049 & .026 & .077 \\
\hline Sexual satisfaction & $\rightarrow$ Relationship satisfaction & $\rightarrow$ & Vitality & & & .143 & .048 & .239 \\
\hline \multicolumn{9}{|c|}{ Indirect associations among within-individuals shifts in specific months } \\
\hline Social isolation stress & $\rightarrow$ Sexual satisfaction & $\rightarrow$ & Irritation with partner & & & .026 & .014 & .040 \\
\hline Social isolation stress & $\rightarrow$ Sexual satisfaction & $\rightarrow$ & Vitality & & & -.014 & -.025 & -.006 \\
\hline Social isolation stress & $\rightarrow$ Sexual satisfaction & $\rightarrow$ & Relationship satisfaction & & & -.052 & -.076 & -.031 \\
\hline Social isolation stress & $\rightarrow$ Sexual satisfaction & $\rightarrow$ & Relationship satisfaction & $\rightarrow$ & Depressive symptoms & .006 & .002 & .011 \\
\hline Social isolation stress & $\rightarrow$ Sexual satisfaction & $\rightarrow$ & Relationship satisfaction & $\rightarrow$ & Vitality & -.007 & -.012 & -.003 \\
\hline
\end{tabular}

Asymmetric confidence intervals for the indirect effects were estimated using the Rmediation online tool (Tofigi \& MacKinnon, 2011). Rel $=$ relationship;

conceptualized as business partners, roommates, lovers, close friends and confidants. We would argue that from a family systems lens, each of those represent potentially distinct systems within the larger relationship. Consistent with the current findings and previous work supporting the spillover hypothesis (e.g., Sturge-Apple et al., 2006), disruptions or conflict within one of those systems can rapidly influence couples' functioning in other systems. For example, conflict in the bedroom (i.e., poorer sexual functioning) could spillover into conflict over chores (affecting the roommate system), and drops in the support a couple provides one another (affecting the friendship system). The current study applied Family Systems Theory by examining sexual, romantic, and individual functioning as key systems within primary relationships. Consistent with Family Systems theory, the results suggested that external stressors from the COVID19 pandemic indeed impacted all three of those systems across time, and suggested possible mechanistic paths for how those systems were interrelated. Future work in couples could therefore extend the current findings by using Family Systems theory as a conceptual framework for modeling the inter-relatedness of various dyadic processes.

There are Different Forms of COVID-19 Related Stress Although the two forms of COVID-19 related stress examined in the current study (i.e., COVID-19 concerns and social isolation stress) are by no means the only forms of stress individuals experienced during the pandemic, distinct patterns of results emerged for these two forms of stress. Specifically, COVID-19 related health concerns were significantly predictive at a between-person level across the 6 months. Greater overall levels of COVID-19 concerns across the 6 months predicted poorer sexual functioning (i.e., more orgasmic difficulties), poorer romantic functioning (i.e., greater irritability with partner), and poorer individual functioning (i.e., more depressive symptoms, lower vitality) across the 6 months. These findings are consistent with previous research indicating chronic health stress to be linked to greater depressive symptoms (e.g., Dalton et al., 2016) even 2 to 5 years later (e.g., Raposa et al., 2014), highlighting the broader links between external stress and lowered relationship functioning (see Randall \& Bodenmann, 2017 for a review) and suggesting that higher COVID-19 related stress is linked to lower vitality (e.g., Peltz et al., 2020), less frequent sexual activity and affection (e.g., Luetke et al., 2020), and lower sexual satisfaction (e.g., Schmid et al., 2021). It is notable that after controlling for stable levels of COVID-19 related stress across the 6 months of the study, shifts in COVID-19 concerns within individuals within specific months failed to emerge as a significant predictor of corresponding shifts in sexual, romantic, or individual functioning. This begins to suggest that the COVID-19 health concerns assessed in the current study might reflect more chronic and stable concerns (e.g., COPD, diabetes, or some other comorbidity in oneself or in a loved one) which would be likely to impact an individual's reactions to the pandemic in a more global manner, raising the personal relevance of the pandemic throughout the 6 months.

In contrast, current findings suggested that the stress associated with the social isolation impacted individuals both in its stable between-person differences across the 6 months, as well as through within-person fluctuations in specific months of the pandemic. Thus, this form of COVID-19 related stress demonstrated both stable trait-like properties (i.e., predictive between-person differences), as well as dynamic statelike properties (i.e., predictive within-person change across time), highlighting how both forms of differences helped to shape sexual, romantic, and individual functioning across the first 6-7 months of the pandemic in the US. These findings are consistent with previous research suggesting that greater social isolation during the COVID-19 pandemic is linked to lower vitality and psychological adjustment (e.g., Arslan, 2021), and greater strain on family relationships (e.g., Evans et al., 2020). 
Extending these patterns beyond the context of the current pandemic, previous work has linked social isolation and social disconnectedness to poorer individual and relationship health, specifically demonstrating social isolation to predict not only greater depressive symptoms or diagnoses (e.g., Ge et al., 2017; Matthews et al., 2016; Suwinyattichaiporn \& Johnson, 2020) and lower vitality (e.g., van Steenbergen et al., 2015), but also greater physical and verbal aggression towards a romantic partner (e.g., Stets, 1991). To that effect, a randomized clinical trial investigating the effectiveness of a social isolation prevention program for older adults has indicated a significant increase in life satisfaction 1 and 6 months post-program among those who received treatment, whereas those within the control group saw no change (Saito et al., 2012). The current study therefore builds on previous findings not only by demonstrating the impact of COVID-19 related social isolation on sexual and romantic functioning, and by highlighting sexual and romantic functioning as possible mechanisms, but also by distinguishing the betweenperson and within-person associations of social isolation.

Exploring Other Forms of COVID-19 Related Stress. Although the two forms of COVID-19 related stress examined in the current study yielded distinct and meaningful results, future work could extend the current findings by examining additional forms of stress that individuals might experience during a pandemic. For example, it could also be useful to examine how financial/employment instability or the stress of new parenting demands impacted various forms of interpersonal and individual functioning during the pandemic. The current results begin to suggest that those alternative forms of stress could very likely offer their own predictive patterns within the Family Systems Theory framework.

Sexual and Relationship Satisfaction Emerged as Key Mechanisms. At the within-person level, sexual satisfaction and relationship satisfaction significantly mediated the association between social isolation stress and individual functioning. Spikes in social isolation stress shaped corresponding decreases in sexual satisfaction and relationship satisfaction, which then let to more depressive symptoms and lower vitality. Thus, the adverse impact of social isolation on sexual and romantic functioning served to intensify the direct associations between social isolation stress and poorer individual functioning. These findings are consistent with spillover effects (e.g., Sturge-Apple et al., 2006) as conceptualized within Family Systems Theory (Broderick, 1993; Minuchin, 1985) as well as other research indicating links between greater sexual functioning and mental health (e.g., Costa \& Brody, 2012; Levin, 2007; Palmore, 1982). The current findings build on previous research by examining associations between acute stress, sexual functioning, relationship functioning, and individual well-being within a broader multivariate, conceptual framework evaluated across time. This allowed us to directly model modeling how pandemic-related stress adversely impacted individual wellbeing through a cascade of lower sexual satisfaction and correspondingly lower relationship satisfaction. These findings may illuminate possible points of intervention, as the results suggested that partnered individuals with robust levels of sexual and relationship satisfaction would have experienced some protection from the adverse impact of social isolation stress across time via the direct and indirect links from those mechanisms to individual outcomes.

Stable Difficulties with Orgasms as a Mechanism. Higher chronic levels of COVID-19 stress across the 6 months of the study were directly linked to higher chronic levels of irritability within relationships. That association was also strengthened by chronic COVID-19 stress predicting chronic difficulties with orgasms, which in turn predicted greater irritability across the 6 months of the study. Thus, stable between-person differences in orgasm difficulties served as a potential mechanism more tightly linking those variables. These findings are consistent with previous work indicating that sexual dysfunctions have been linked to negative conflict in relationships (e.g., Metz \& Epstein, 2002). Extending these quantitative findings, qualitative analyses of interviews of 20 Iranian women highlighted conflict and annoyance with a partner as key obstacles to reaching orgasm during sexual activity (Nekoolaltak et al., 2017). Those qualitative findings dovetail the current findings, suggesting that the links between orgasm difficulties and irritability within relationships might be transactional in nature, reciprocally influencing one another across time. The current findings further suggested that chronic levels of orgasm difficulties across the 6 months served to intensify the direct association between higher chronic COVID-19 stress and greater depressive symptoms across the 6 months of the study. This is consistent with previous findings linking orgasm difficulties to greater depressive symptoms (e.g., Castellini et al., 2010; Forbes et al., 2016; Shifren et al., 2008). The current study contextualizes those previous findings within the Family Systems Theory, helping to highlight the potential mediating roles of difficulties with orgasms.

Sexual Satisfaction as a Source of Resilience. One of the strongest indirect paths to emerge in the model was at level 2 (the level of stable between-person differences on average levels of each construct across the 6 months of the study) and involved sexual satisfaction as a predictor. Contrary to our expectations, stable levels of COVID-19 related stress across the first 6 months of the pandemic in the United States were not significantly predictive of corresponding stable levels of sexual satisfaction. This would suggest that at an aggregate level (i.e., across all partnered participants) individuals' overall levels of sexual satisfaction were on average unaffected by COVID-19 related stress. These results are consistent with the mixed findings concerning the impact of the COVID-19 pandemic on sexual health (e.g., Panzeri et al., 2020). They 
suggest that for individuals in relationships with high levels of sexual functioning, their sexual health could serve as a source of resilience whereas for individuals in relationships with low levels of sexual functioning, their sexual difficulties could serve as a source of risk. Consistent with this, stably high levels of sexual satisfaction across the six months of the study were not only directly predictive of higher stable levels of vitality, but were also strongly linked to higher stable levels of relationship satisfaction, which in turn predicted even higher vitality across the first six months of the pandemic. Thus, sexual satisfaction emerged as a key factor fueling well-being in the first six months of the COVID-19 pandemic, highlighting its key roles in individual health and well-being (e.g., Costa \& Brody, 2012; Levin, 2007; Palmore, 1982).

Communicating Affection as a Source of Resilience. An unexpected finding emerged suggesting that stable betweenperson differences in COVID-19 health concern stress across the 6 months predicted greater stable levels of communicating affection to loved ones across those 6 months. Thus, fears over contracting COVID-19 and potentially getting sick themselves or losing loved ones seemed to help individuals prioritize their close relationships, spurring them on to share their love and affection with the people around them. These findings are consistent with the literature suggesting that connection with others is a fundamental human need (e.g., Baumeister \& Leary, 1995) and that individuals are driven to affiliate (i.e., tend and befriend others) under conditions of stress (e.g., Taylor, 2002, 2006). In fact, the communication of affection has been shown to be an effective coping mechanism as it has been shown to help buffer couples from the strain of a new child on their romantic relationships (Shapiro et al., 2000). The current findings are also consistent with recent theoretical assertions (based on applying the Family Systems Theory to the pandemic) that adaptive relationship processes could likely buffer the link between pandemic stress and well-being (Pietromonaco \& Overall, 2021). The current study therefore builds on previous work by demonstrating affiliation (via the communication of affection) as an effective coping mechanism during the COVID-19 pandemic.

\section{Limitations and Future Directions}

The current study builds on a body of predominantly crosssectional work by analyzing longitudinal data collected from a robust sample during a critical time in history, while the COVID-19 pandemic began to spread through the United States. By drawing key constructs from multiple fields of study (individual psychopathology, positive psychology, sexual health, and couples and marital research) and integrating them within a Family Systems Theory framework, the current study also sought to provide a more nuanced understanding of the impact of the COVID-19 pandemic on the lives of individuals in the US. The results underscored the importance of sexual and romantic functioning as potential mechanisms linking COVID-19 related stress to individual well-being, thereby highlighting potential sources of resilience and risk.

Despite these strengths, a number of issues limit the current findings. First, the current data was analyzed in a sample predominantly made up of white individuals and women. Although our large sample size provided 419 men and 231 non-white individuals, future studies with more diverse samples are needed to explore the generalizability of the current findings. Second, the study relied solely on self-report data, raising concerns of responses being limited by: possible lack of insight (i.e., being unaware or out of touch with one's own behavior and/or internal experiences and therefore having a difficult time accurately completing self-report scales), potential reporting biases, and social desirability. To address this, future studies could collect data from multiple informants (i.e., including data from participants' friends, family members, and/ or romantic partners), collect observational data, and assess possible response biases to ensure the results remain consistent when such limitations are addressed. Extending this point, although social desirability has been shown to be somewhat less of a problem for survey data collected online (Richman et al., 1999), the current study did not specifically assess levels of socially desirable responding. Thus, future studies could examine this as a possible source of additional variance in models of functioning during pandemics. Third, the current sample consisted of data from only one partner from each couple, providing a one-sided perspective on each relationship. Future research would benefit from collecting data from both romantic partners, providing a more balanced view of the relationships and enabling dyadic analyses. Fourth, although this study examined two specific forms of COVID-19 related stress, the pandemic and associated public health measures created a wide range of chaos and upheaval to daily life across the globe. Thus, future work could extend these findings and the Family Systems Theory by examining other sources of stress and upheaval as potential predictors. Fifth, recruitment for the current study just as the pandemic was beginning to draw national attention in the United States and as a result, the study did not include a truly pre-pandemic baseline for a majority of the participants and the baseline survey did not include measures of COVID-19 related stress. Thus, although the current findings speak to how the constructs examined fluctuated in response to one another across time during a pandemic, they do not provide insights on how those dynamics might have changed in comparison to pre-pandemic functioning. Future work could seek to examine components of this same model once the pandemic has largely resolved to determine the degree to which the associations between various relationship systems (i.e., sexual, romantic, and individual functioning) were specific to the context of the pandemic or were more broadly representative of relationship 
dynamics. Sixth, although the study title, "The Finding Pleasure in Sex Study" effectively communicated to prospective participants the contents of the survey, that title could have inadvertently encouraged the participation of people with more sex-positive attitudes and with greater comfort discussing sexuality and sexual behavior. Balancing this concern, for a majority of the respondents (i.e., those recruited after the midMarch 2020 modification of the study), the bulleted list presenting the details of the study immediately following that title included a bullet referring to tracking the effects of COVID-19 across time: "Track the impact of COVID-19 on the lives of individuals" thereby increasing the relevance of the study to a broader range of individuals. Future work could explore the current model using a less sexualized title to help reduce the risk of biasing the sample. Extending that concern, the study made use of a convenience sample, thereby introducing possible selection bias. Future work could use random sampling techniques to help ensure the representativeness of the final sample. Seventh, several of the measures used in the current study were developed by the authors given the tight timeframe for starting a COVID-19 focused project. Future work could therefore extend research on this model and these hypotheses by examining them using existing measures that are well validated within the current literature. Eighth, although the analyses demonstrated reasonable model invariance across multiple demographic groups and controlled for parent and employment status as possible confounds, it is still likely that factors not included in the current model might have also helped to shape the sexual, romantic, and individual functioning of individuals during the first six months of the pandemic. Thus, future work could extend the current findings by examining a broader set of potential confounds. Extending that point, despite highlighting sexual functioning and romantic functioning as key factors influencing the impact of the pandemic on people in relationships, those are not the only possible sources of risk and resilience for individuals. Thus, future work could extend the current work by examining a broader set of risk/resilience factors including: (1) background factors and traits (e.g., sex positivity/erotophilia, trait negativity, optimism, grit, mindfulness, psychological flexibility, adult attachment), (2) other relationship subsystems (e.g., friendship/support/intimacy, business/ financial, roommate/chores/distribution of labor), and (3) other external factors (e.g., work stress, conflict or stress within individuals' extended family/friend network). Ninth, although over 600 respondents provided follow-up data, roughly $40 \%$ of our baseline parents did not complete any follow-up surveys. This level of attrition is not uncommon for online studies involving no monetary compensation (e.g., Daks et al., 2021) and was likely due in part to the upheaval of the COVID-19 pandemic on the lives of individuals. Strengthening this concern, although attrition analyses failed to find differences on levels of income or vitality at baseline, those analyses suggested that the respondents providing follow-up data were somewhat more likely to be white, heterosexual, women, engaged/married, have graduate degrees, and who were slightly older with slightly fewer depressive symptoms. Thus, despite having roughly 1800 distinct longitudinal assessments from over 600 respondents supporting our models (above and beyond the baseline assessments from all respondents), future longitudinal studies could extend the current study by seeking funding to help reduce attrition, thereby ensuring that the subtle attrition differences within the current study are not unduly influencing the results. Tenth, although a majority of the sample were in fairly longterm relationships ( $63 \%$ together for $3+$ years, $71 \%$ together for $2+$ years), $18 \%$ of the individuals had been in their relationships for less than a year and $10 \%$ had been with their partners less than 6 months, suggesting fairly high levels of heterogeneity in the relationships represented in the sample. Fortunately, the model results remained invariant across relationship stages and across cohabiting vs non-cohabiting couples, suggesting that the results generalized across the wide range of relationship stages included in the study, thereby reducing concerns about relationship heterogeneity. Future work could serve to replicate the current findings by examining similar models within more specific stages of romantic relationships. Despite these limitations, the results offer compelling evidence suggesting sexual and romantic functioning as buffering mediators between COVID-19 related stress and poor individual functioning.

\section{Conclusions}

The current study drew an array of constructs from multiple literatures, measured them in a large online sample on a monthly basis across the first 7-8 months of the COVID-19 pandemic in the US, and then ran multilevel models to examine their associations across time within a Family Systems Theory framework. At the level of stable between-person differences across the study, the findings suggested that: (1) individuals in rewarding relationships with satisfying sex lives were likely to have made it through the first 6 months of the pandemic with greater vitality and fewer depressive symptoms (highlighting those variables as sources of resilience), (2) stable levels of stress from social isolation prompted individuals to express their love and affection for the people closest to them (highlighting the need for social connection), and (3) stable difficulties with orgasms were linked to individuals being more irritable and cranky with their partners across the 6 months of the study and experiencing greater depressive symptoms (highlighting that as a source of risk). In contrast, at the level of dynamic change on a month-to-month basis, the findings revealed how aspects of sexual and romantic functioning might act as mechanisms linking various forms of COVID-19 related stress to individual well-being. Thus, although elevations in social isolation stress within specific months demonstrated direct links to drops individual functioning in those same months, 
that negative association was further reinforced by its links to corresponding drops in sexual and relationship satisfaction (highlighting key warning signs and potential points for clinical intervention). Thus, these findings not only provide a more nuanced understanding of the impact of COVID-19 related stress on individual functioning, but they also offer relevant insights for clinicians and therapists working with individuals and couples during periods of acute stress like the COVID-19 pandemic.

Author Contributions RR and AP designed the study together and developed the study materials. RR obtained IRB approval and programmed the survey online. RR and AP recruited the sample, cleaned the data, and ran the analyses together. RR developed the tables and figures while AP wrote the first draft of the manuscript. Both authors then copy edited the manuscript and are responsible for all of its content.

Funding There are no funding sources to report.

Data Availability The IRB materials, study materials, SPSS syntax, Mplus syntax and output, and data for this manuscript will be made available on the osf.io website.

\section{Declarations}

Conflict of interest Neither author has any financial conflicts of interest to report for this project or this manuscript.

Informed Consent The study was evaluated and approved as a minimal risk study by a university IRB. Participants were presented with an information sheet on the first page of the survey to obtain informed consent, and the study was conducted conforming to all ethical guidelines.

\section{References}

Arons, M. M., Hatfield, K. M., Reddy, S. C., Kimball, A., James, A., Jacobs, J. R., Taylor, J., Spicer, K., Bardossy, A. C., Oakley, L. P., Tanwar, S., Dyal, J. W., Harney, J., Chisty, Z., Bell, J. M., Methner, M., Paul, P., Carlson, C. M., McLaughlin Jernigan, J. A., for the Public Health-Seattle and King County and CDC COVID-19 Investigation Team. (2020). Presymptomatic SARS-CoV-2 infections and transmission in a skilled nursing facility. New England Journal of Medicine, 382, 2081-2090. https://doi.org/10.1056/ NEJMoa2008457

Arslan, G. (2021). Loneliness, college belongingness, subjective vitality, and psychological adjustment during coronavirus pandemic: Development of the college belongingness questionnaire. Journal of Positive School Psychology, 5. Retrieved from https://journ alppw.com/index.php/JPPW/article/view/240

Baumeister, R. F., Catanese, K. R., \& Vohs, K. D. (2001). Is there a gender difference in strength of sex drive? Theoretical views, conceptual distinctions, and a review of relevant evidence. Personality and Social Psychology Review, 5, 242-273. https://doi. org/10.1207/S15327957PSPR0503_5

Baumeister, R. F., \& Leary, M. R. (1995). The need to belong: Desire for interpersonal attachments as a fundamental human motivation. Psychological Bulletin, 117, 497-529. https://doi.org/10. 4324/9781351153683-3
Broderick, C. B. (1993). Understanding family process: Basics of family systems theory. Sage.

Brody, S., \& Costa, R. M. (2009). Satisfaction (sexual, life, relationship, and mental health) is associated directly with penile-vaginal intercourse, but inversely with other sexual behavior frequencies. Journal of Sexual Medicine, 6, 1947-1954. https://doi.org/10. 1111/j.1743-6109.2009.01303.x

Brooks, S. K., Webster, R. K., Smith, L. E., Woodland, L., Wessely, S., Greenberg, N., \& Rubin, G. J. (2020). The psychological impact of quarantine and how to reduce it: Rapid review of the evidence. Lancet, 395, 912-920. https://doi.org/10.1016/S0140-6736(20) 30460-8

Buczak-Stec, E., König, H. H., \& Hajek, A. (2021). Sexual satisfaction of middle-aged and older adults: Longitudinal findings from a nationally representative sample. Age and Ageing, 50, 559-564. https://doi.org/10.1093/ageing/afaa161

Bueno-Notivol, J., Gracia-García, P., Olaya, B., Lasheras, I., LópezAntón, R., \& Santabárbara, J. (2021). Prevalence of depression during the COVID-19 outbreak: A meta-analysis of communitybased studies. International Journal of Clinical and Health Psychology, 21, 100196. https://doi.org/10.1016/j.ijchp.2020.07.007

Castellini, G., Mannucci, E., Mazzei, C., Lo Sauro, C., Faravelli, C., Rotella, C. M., Maggi, M., \& Ricca, V. (2010). Sexual function in obese women with and without binge eating disorder. Journal of Sexual Medicine, 7, 3969-3978. https://doi.org/10.1111/j.17436109.2010.01990.x

Chang, S. R., Yang, C. F., \& Chen, K. H. (2019). Relationships between body image, sexual dysfunction, and health-related quality of life among middle-aged women: A cross-sectional study. Maturitas, 126, 45-50. https://doi.org/10.1016/j.maturitas.2019.04.218

Costa, R. M., \& Brody, S. (2007). Women's relationship quality is associated with specifically penile-vaginal intercourse orgasm and frequency. Journal of Sex \& Marital Therapy, 33, 319-327. https:// doi.org/10.1080/00926230701385548

Costa, R. M., \& Brody, S. (2012). Greater resting heart rate variability is associated with orgasms through penile-vaginal intercourse, but not with orgasms from other sources. Journal of Sexual Medicine, 9, 188-197. https://doi.org/10.1111/j.1743-6109.2011.02541.x

Crasta, D., Daks, J. S., \& Rogge, R. D. (2020). Modeling suicide risk among parents during the COVID-19 pandemic: Psychological inflexibility exacerbates the impact of COVID-19 stressors on interpersonal risk factors for suicide. Journal of Contextual Behavioral Science, 18, 117-127. https://doi.org/10.1016/j.jcbs. 2020.09.003

Daks, J. S., Peltz, J. S., \& Rogge, R. D. (2020). Psychological flexibility and inflexibility as sources of resiliency and risk during a pandemic: Modeling the cascade of COVID-19 stress on family systems with a contextual behavioral science lens. Journal of Contextual Behavioral Science, 18, 16-27. https://doi.org/10.1016/j. jcbs.2020.08.003

Daks, J. S., Rogge, R. D., \& Fincham, F. D. (2021). Distinguishing the correlates of being mindfully vs. mindlessly coupled: Development and validation of the Attentive Awareness in Relationships Scale (AAIRS). Mindfulness, 12, 1361-1376. https://doi.org/10.1007/ s12671-021-01604-w

Dalton, E. D., Hammen, C. L., Brennan, P. A., \& Najman, J. M. (2016). Pathways maintaining physical health problems from childhood to young adulthood: The role of stress and mood. Psychology \& Health, 31, 1255-1271. https://doi.org/10.1080/08870446.2016. 1204448

Diener, E. D., Emmons, R. A., Larsen, R. J., \& Griffin, S. (1985). The Satisfaction with Life Scale. Journal of Personality Assessment, 49, 71-75. https://doi.org/10.1037/t01069-000

Döring, N. (2020). How is the COVID-19 pandemic affecting our sexualities? An overview of the current media narratives and research 
hypotheses [Commentary]. Archives of Sexual Behavior, 49, 27652778. https://doi.org/10.1007/s10508-020-01790-z

Ellsworth, R. M., \& Bailey, D. H. (2013). Human female orgasm as evolved signal: A test of two hypotheses. Archives of Sexual Behavior, 42, 1545-1554. https://doi.org/10.1007/s 10508-013-0152-7

Ettman, C. K., Abdalla, S. M., Cohen, G. H., Sampson, L., Vivier, P. M., \& Galea, S. (2020). Prevalence of depression symptoms in US adults before and during the COVID-19 pandemic. JAMA Network Open, 3, e2019686-e2019686. https://doi.org/10.1001/jamanetwor kopen.2020.19686

Evans, S., Mikocka-Walus, A., Klas, A., Olive, L., Sciberras, E., Karantzas, G., \& Westrupp, E. M. (2020). From 'It has stopped our lives' to 'Spending more time together has strengthened bonds': The varied experiences of Australian families during COVID-19.' Frontiers in Psychology, 11, 1-13. https://doi.org/10.3389/fpsyg. 2020.588667

Falconier, M. K., Nussbeck, F., Bodenmann, G., Schneider, H., \& Bradbury, T. (2015). Stress from daily hassles in couples: Its effects on intradyadic stress, relationship satisfaction, and physical and psychological well-being. Journal of Marital and Family Therapy, 41, 221-235. https://doi.org/10.1111/jmft.12073

Fallis, E. E., Rehman, U. S., Woody, E. Z., \& Purdon, C. (2016). The longitudinal association of relationship satisfaction and sexual satisfaction in long-term relationships. Journal of Family Psychology, 30, 822-831. https://doi.org/10.1037/fam0000205

Floyd, K. (2002). Human affection exchange: V. Attributes of the highly affectionate. Communication Quarterly, 50, 135-152. https://doi. org/10.1080/01463370209385653

Forbes, M. K., Baillie, A. J., \& Schniering, C. A. (2016). Should sexual problems be included in the internalizing spectrum? A comparison of dimensional and categorical models. Journal of Sex \& Marital Therapy, 42, 70-90. https://doi.org/10.1080/0092623X.2014. 99692

Frederick, D. A., John, H. K. S., Garcia, J. R., \& Lloyd, E. A. (2018). Differences in orgasm frequency among gay, lesbian, bisexual, and heterosexual men and women in a US national sample. Archives of Sexual Behavior, 47(1), 273-288.

Funk, J. L., \& Rogge, R. D. (2007). Testing the ruler with item response theory: Increasing precision of measurement for relationship satisfaction with the couples satisfaction index. Journal of Family Psychology, 21, 572-583. https://doi.org/10.1037/0893-3200.21.4.572

Garcia, J. R., Lloyd, E. A., Wallen, K., \& Fisher, H. E. (2014). Variation in orgasm occurrence by sexual orientation in a sample of US singles. Journal of Sexual Medicine, 11, 2645-2652. https://doi. org/10.1111/jsm.12669

Ge, L., Yap, C. W., Ong, R., \& Heng, B. H. (2017). Social isolation, loneliness and their relationships with depressive symptoms: A population-based study. PLOS ONE, 12(8), e0182145. https://doi. org/10.1371/journal.pone.0182145

Haavio-Mannila, E., \& Kontula, O. (1997). Correlates of increased sexual satisfaction. Archives of Sexual Behavior, 26, 399-419. https://doi.org/10.1023/A:1024591318836

Harper, J. M., Schaalje, B. G., \& Sandberg, J. G. (2000). Daily hassles, intimacy, and marital quality in later life marriages. American Journal of Family Therapy, 28, 1-18. https://doi.org/10.1080/ 019261800261770

Hollist, C. S., Miller, R. B., Falceto, O. G., \& Fernandes, C. L. C. (2007). Marital satisfaction and depression: A replication of the marital discord model in a Latino sample. Family Process, 46, 485-498. https://doi.org/10.1111/j.1545-5300.2007.00227.x

Hurlbert, D. F., Apt, C., \& Rabehl, S. M. (1993). Key variables to understanding female sexual satisfaction: An examination of women in nondistressed marriages. Journal of Sex \& Marital Therapy, 19, 154-165. https://doi.org/10.1080/00926239308404899

Karagöz, M. A., Gül, A., Borg, C., Erihan, İB., Uslu, M., Ezer, M., Erbağci, A., Çatak, B., \& Bağcı̆ğlu, M. (2020). Influence of
COVID-19 pandemic on sexuality: A cross-sectional study among couples in Turkey. International Journal of Impotence Research. https://doi.org/10.1038/s41443-020-00378-4

Karney, B. R., \& Bradbury, T. N. (1995). The longitudinal course of marital quality and stability: A review of theory, methods, and research. Psychological Bulletin, 118, 3-34. https://doi.org/10. 1037/0033-2909.118.1.3

Ko, N. Y., Lu, W. H., Chen, Y. L., Li, D. J., Chang, Y. P., Wu, C. F., Wang, P. W., \& Yen, C. F. (2020). Changes in sex life among people in Taiwan during the COVID-19 pandemic: The roles of risk perception, general anxiety, and demographic characteristics. International Journal of Environmental Research and Public Health, 17, 5822. https://doi.org/10.3390/ijerph17165822

Kucharski, A. J., Russell, T. W., Diamond, C., Liu, Y., Edmunds, J., Funk, S., \& Davies, N. (2020). Early dynamics of transmission and control of COVID-19: A mathematical modelling study. Lancet Infectious Diseases, 20, 553-558. https://doi.org/10.1016/S14733099(20)30144-4

Laan, E., \& Rellini, A. H. (2012). Can we treat anorgasmia in women? The challenge to experiencing pleasure. Sexual and Relationship Therapy, 26, 329-341. https://doi.org/10.1080/14681994.2011. 649691

Laumann, E. O., Gangon, J. H., Michael, R. T., \& Michaels, T. (2000). The social organization of sexuality: Sexual practices in the United States. University of Chicago Press.

Leach, L. S., Butterworth, P., Olesen, S. C., \& Mackinnon, A. (2013). Relationship quality and levels of depression and anxiety in a large population-based survey. Social Psychiatry and Psychiatric Epidemiology, 48, 417-425. https://doi.org/10.1007/s00127-012-0559-9

Legate, N., \& Rogge, R. D. (2019). Identifying basic classes of sexual orientation with latent profile analysis: Developing the multivariate sexual orientation classification system. Archives of Sexual Behavior, 48, 1403-1422. https://doi.org/10.1007/s10508-018-1336-y

Levin, R. J. (2007). Sexual activity, health and well-being: The beneficial roles of coitus and masturbation. Sexual and Relationship Therapy, 22, 135-148. https://doi.org/10.1080/146819906011491 97

Li, G., Tang, D., Song, B., Wang, C., Qunshan, S., Xu, C., Geng, H., Wu, H., He, X., \& Cao, Y. (2020). Impact of the COVID-19 pandemic on partner relationships and sexual and reproductive health: Cross-sectional, online survey study. Journal of Medical Internet Research, 22, e20961. https://doi.org/10.2196/20961

Luetke, M., Hensel, D., Herbenick, D., \& Rosenberg, M. (2020). Romantic relationship conflict due to the COVID-19 pandemic and changes in intimate and sexual behaviors in a nationally representative sample of American adults. Journal of Sex \& Marital Therapy, 46, 747-762. https://doi.org/10.1080/0092623X.2020.1810185

Matthews, T., Danese, A., Wertz, J., Odgers, C. L., Ambler, A., Moffitt, T. E., \& Arseneault, L. (2016). Social isolation, loneliness and depression in young adulthood: A behavioural genetic analysis. Social Psychiatry and Psychiatric Epidemiology, 51(3), 339-348. https://doi.org/10.1007/s00127-016-1178-7

McNulty, J. K., Wenner, C. A., \& Fisher, T. D. (2016). Longitudinal associations among relationship satisfaction, sexual satisfaction, and frequency of sex in early marriage. Archives of Sexual Behavior, 45, 85-97. https://doi.org/10.1007/s10508-014-0444-6

Metz, M. E., \& Epstein, N. (2002). Assessing the role of relationship conflict in sexual dysfunction. Journal of Sex \& Marital Therapy, 28, 139-164. https://doi.org/10.1080/00926230252851889

Minuchin, P. (1985). Families and individual development: Provocations from the field of family therapy. Child Development, 56, 289-302. https://doi.org/10.2307/1129720

Nekoolaltak, M., Keshavarz, Z., Simbar, M., Nazari, A. M., \& Baghestani, A. R. (2017). Women's orgasm obstacles: A qualitative study. International Journal of Reproductive BioMedicine, 15, 479-490. https://doi.org/10.29252/ijrm.15.8.479 
New York Times. (2021, May). Coronavirus world map: Tracking the global outbreak. https://www.nytimes.com/interactive/2020/ world/coronavirus-maps.html

Palmore, E. B. (1982). Predictors of the longevity difference: A 25-year follow-up. The Gerontologist, 22, 513-518. https://doi.org/10. 1093/geront/22.6.513

Panzeri, M., Ferrucci, R., Cozza, A., \& Fontanesi, L. (2020). Changes in sexuality and quality of couple relationship during the COVID-19 lockdown. Frontiers in Psychology, 11, 1-15. https://doi.org/10. 3389/fpsyg.2020.565823

Peltz, J. S., Daks, J. S., \& Rogge, R. D. (2020). Mediators of the association between COVID-19-related stressors and parents' psychological flexibility and inflexibility: The roles of perceived sleep quality and energy. Journal of Contextual Behavioral Science, 17, 168-176. https://doi.org/10.1016/j.jcbs.2020.07.001

Peplau, L. A., \& Fingerhut, A. W. (2007). The close relationships of lesbians and gay men. Annual Review of Psychology, 58, 405-424. https://doi.org/10.1146/annurev.psych.58.110405.085701

Pieh, C., O'Rourke, T., Budimir, S., \& Probst, T. (2020). Relationship quality and mental health during COVID-19 lockdown. PLoS ONE, 15, e0238906. https://doi.org/10.1371/journal.pone.0238906

Pietromonaco, P. R., \& Overall, N. C. (2021). Applying relationship science to evaluate how the COVID-19 pandemic may impact couples' relationships. American Psychologist, 76, 438-450. https:// doi.org/10.1037/amp0000714

Preacher, K. J., Zyphur, M. J., \& Zhang, Z. (2010). A general multilevel SEM framework for assessing multilevel mediation. Psychological Methods, 15(3), 209-233. https://doi.org/10.1037/a0020141

Randall, A. K., \& Bodenmann, G. (2017). Stress and its associations with relationship satisfaction. Current Opinion in Psychology, 13, 96-106. https://doi.org/10.1016/j.copsyc.2016.05.010

Raposa, E., Hammen, C., Brennan, P., \& Najman, J. (2014). The longterm effects of maternal depression: Early childhood physical health as a pathway to offspring depression. Journal of Adolescent Health, 54, 88-93. https://doi.org/10.1016/j.jadohealth.2013. 07.038

Richman, W. L., Kiesler, S., Weisband, S., \& Drasgow, F. (1999). A meta-analytic study of social desirability distortion in computeradministered questionnaires, traditional questionnaires, and interviews. Journal of Applied Psychology, 84, 754-775. https://doi. org/10.1037/0021-9010.84.5.754

Rosen, C., Brown, J., Heiman, S., Leiblum, C., Meston, R., Shabsigh, D., Ferguson, R., \& D'Agostino, R. (2000). The Female Sexual Function Index (FSFI): A multidimensional self-report instrument for the assessment of female sexual function. Journal of Sex \& Marital Therapy, 26, 191-208. https://doi.org/10.1080/00926 2300278597

Ryan, R. M., \& Frederick, C. (1997). On energy, personality, and health: Subjective vitality as a dynamic reflection of well-being. Journal of Personality, 65(3), 529-565. https://doi.org/10.1111/j.1467-6494. 1997.tb00326.x

Saito, T., Kai, I., \& Takizawa, A. (2012). Effects of a program to prevent social isolation on loneliness, depression, and subjective wellbeing of older adults: A randomized trial among older migrants in Japan. Archives of Gerontology and Geriatrics, 55, 539-547. https://doi.org/10.1016/j.archger.2012.04.002

Salari, N., Hosseinian-Far, A., Jalali, R., Vaisi-Raygani, A., Rasoulpoor, S., Mohammadi, M., Rasoulpoor, S., \& Khaledi-Paveh, B. (2020). Prevalence of stress, anxiety, depression among the general population during the COVID-19 pandemic: A systematic review and meta-analysis. Globalization and Health, 16, 1-11. https://doi.org/ 10.1186/s12992-020-00589-w

Sarti, C. D., Graziottin, A., Mincigrucci, M., Ricci, E., Chiaffarino, F., Bonaca, S., Becorpi, A., \& Parazzini, F. (2010). Correlates of sexual functioning in Italian menopausal women. Climacteric, 13, 447-456. https://doi.org/10.3109/13697130903491026
Schermerhorn, A. C., \& Cummings, E. M. (2008). Transactional family dynamics: A new framework for conceptualizing family influence processes. Advances in Child Development and Behavior, 36, 187-250. https://doi.org/10.1016/S0065-2407(08)00005-0

Schmid, L., Wörn, J., Hank, K., Sawatzki, B., \& Walper, S. (2021). Changes in employment and relationship satisfaction in times of the COVID-19 pandemic: Evidence from the German family Panel. European Societies, 23. https://doi.org/10.1080/14616696. 2020.1836385

Shapiro, A. F., Gottman, J. M., \& Carrere, S. (2000). The baby and the marriage: Identifying factors that buffer against decline in marital satisfaction after the first baby arrives. Journal of Family Psychology, 14, 59-70. https://doi.org/10.1037/0893-3200.14.1.59

Shaw, A. M., \& Rogge, R. D. (2016). Evaluating and refining the construct of sexual quality with item response theory: Development of the quality of sex inventory. Archives of Sexual Behavior, 45, 249-270. https://doi.org/10.1007/s10508-015-0650-x

Shifren, J. L., Monz, B. U., Russo, P. A., Segreti, A., \& Johannes, C. B. (2008). Sexual problems and distress in United States women: Prevalence and correlates. Obstetrics \& Gynecology, 112, 970978. https://doi.org/10.1097/AOG.0b013e3181898cdb

Sin, N. L., \& Lyubomirsky, S. (2009). Enhancing well-being and alleviating depressive symptoms with positive psychology interventions: A practice-friendly meta-analysis. Journal of Clinical Psychology, 65, 467-487. https://doi.org/10.1002/jclp.20593

Spitzer, R. L., Kroenke, K., Williams, J. B., \& Patient Health Questionnaire Primary Care Study Group. (1999). Validation and utility of a self-report version of PRIME-MD: The PHQ Primary Care Study. Journal of the American Medical Association, 282, 1737 1744. https://doi.org/10.1001/jama.282.18.1737

Stets, J. E. (1991). Cohabiting and marital aggression: The role of social isolation. Journal of Marriage and the Family, 53, 669-680. https://doi.org/10.2307/352742

Sturge-Apple, M. L., Davies, P. T., \& Cummings, E. M. (2006). Impact of hostility and withdrawal in interparental conflict on parental emotional unavailability and children's adjustment difficulties. Child Development, 77, 1623-1641. https://doi.org/10.1111/j. 1467-8624.2006.00963.x

Suwinyattichaiporn, T., \& Johnson, Z. D. (2020). The impact of family and friends social support on Latino/a first-generation college students' perceived stress, depression, and social isolation. Journal of Hispanic Higher Education. https://doi.org/10.1177/15381 92720964922

Tang, F., Liang, J., Zhang, H., Kelifa, M. M., He, Q., \& Wang, P. (2021). COVID-19 related depression and anxiety among quarantined respondents. Psychology \& Health, 36, 164-178. https://doi.org/ 10.1080/08870446.2020.1782410

Taylor, S. E. (2002). The tending instinct: How nurturing is essential to who we are and how we live. Holt.

Taylor, S. E. (2006). Tend and befriend: Biobehavioral bases of affiliation under stress. Current Directions in Psychological Science, 15, 273-277. 10.1111\%2Fj.1467-8721.2006.00451.x

Tofighi, D., \& MacKinnon, D. P. (2011). RMediation: An R package for mediation analysis confidence intervals. Behavior Research Methods, 43, 692-700. https://doi.org/10.3758/s13428-011-0076-х

Van Steenbergen, E., Van Dongen, J. M., Wendel-Vos, G. C. W., Hildebrandt, V. H., \& Strijk, J. E. (2016). Insights into the concept of vitality: Associations with participation and societal costs. European Journal of Public Health, 26, 354-359. https://doi.org/10. 1093/eurpub/ckv194

Watson, D., Clark, L. A., \& Tellegen, A. (1988). Development and validation of brief measures of positive and negative affect: The PANAS scales. Journal of Personality and Social Psychology, 54, 1063-1070. https://doi.org/10.1037/0022-3514.54.6.1063

Watson, D., Weber, K., Assenheimer, J. S., Clark, L. A., Strauss, M. E., \& McCormick, R. A. (1995). Testing a tripartite model: I. 
Evaluating the convergent and discriminant validity of anxiety and depression symptom scales. Journal of Abnormal Psychology, 104, 3-14. https://doi.org/10.1037/0021-843X.104.1.3

Whitton, S. W., \& Kuryluk, A. D. (2012). Relationship satisfaction and depressive symptoms in emerging adults: Cross-sectional associations and moderating effects of relationship characteristics. Journal of Family Psychology, 26, 226-235. https://doi.org/10. 1037/a0027267

Whitton, S. W., \& Whisman, M. A. (2010). Relationship satisfaction instability and depression. Journal of Family Psychology, 24, 791-794. https://doi.org/10.1037/a002173

Zhang, S. X., Wang, Y., Rauch, A., \& Wei, F. (2020a). Unprecedented disruption of lives and work: Health, distress and life satisfaction of working adults in China one month into the COVID-19 outbreak. Psychiatry Research, 288. https://doi.org/10.1016/j.psych res.2020.112958
Zhang, X., Wang, Y., Lyu, H., Zhang, Y., Liu, Y., \& Luo, J. (2020b). The influence of COVID-19 on well-being. PsyArXiv. https://doi. org/10.31234/osf.io/znj7h

Zhou, F., Yu, T., Du, R., Fan, G., Liu, Y., Liu, Z., \& Guan, L. (2020). Clinical course and risk factors for mortality of adult inpatients with COVID-19 in Wuhan, China: A retrospective cohort study. Lancet, 395, 1054-1062. https://doi.org/10.1016/S0140-6736(20) 30566-3

Publisher's Note Springer Nature remains neutral with regard to jurisdictional claims in published maps and institutional affiliations. 